This item was submitted to Loughborough's Research Repository by the author.

Items in Figshare are protected by copyright, with all rights reserved, unless otherwise indicated.

\title{
Deformed quantum Calogero-Moser problems and Lie superalgebras
}

PLEASE CITE THE PUBLISHED VERSION

LICENCE

CC BY-NC-ND 4.0

\section{REPOSITORY RECORD}

Sergeev, A.N., and A.P. Veselov. 2019. "Deformed Quantum Calogero-moser Problems and Lie Superalgebras". figshare. https://hdl.handle.net/2134/312. 


\title{
Deformed quantum Calogero-Moser problems and Lie superalgebras
}

\author{
A.N. Sergeev ${ }^{\dagger, \ddagger}$ and A.P. Veselov ${ }^{\ddagger, \star}$ \\ $\dagger$ Balakovo Institute of Technology and Control, Balakovo,413800, Russia \\ $\ddagger$ Department of Mathematical Sciences, Loughborough University, \\ Loughborough, LE11 3TU, UK \\ * Landau Institute for Theoretical Physics, Kosygina 2, Moscow, 117940, \\ Russia \\ E-mail addresses: A.N. Sergeev@lboro.ac.uk, A.P.Veselov@lboro.ac.uk
}

\begin{abstract}
The deformed quantum Calogero-Moser-Sutherland problems related to the root systems of the contragredient Lie superalgebras are introduced. The construction is based on the notion of the generalized root systems suggested by $\mathrm{V}$. Serganova. For the classical series a recurrent formula for the quantum integrals is found, which implies the integrability of these problems. The corresponding algebras of the quantum integrals are investigated, the explicit formulas for their Poincare series for generic values of the deformation parameter are presented.
\end{abstract}

\section{INTRODUCTION}

Calogero-Moser-Sutherland (CMS) problem in its original form [1],[2] describes the particles on the line pairwise interacting with the potential

$$
U\left(x_{1}, \ldots, x_{n}\right)=\sum_{1 \leq i<j \leq n} \frac{g^{2} \omega^{2}}{\sin ^{2} \omega\left(x_{i}-x_{j}\right)}
$$

In the limit $\omega \rightarrow 0$ one has the rational potential

$$
U\left(x_{1}, \ldots, x_{n}\right)=\sum_{1 \leq i<j \leq n} \frac{g^{2}}{\left(x_{i}-x_{j}\right)^{2}}
$$

Olshanetsky and Perelomov [3] proposed a generalization of these problems related to any root system. Corresponding quantum Hamiltonian has a form

$$
L=-\Delta+\sum_{\alpha \in R_{+}} \frac{m_{\alpha}\left(m_{\alpha}+2 m_{2 \alpha}+1\right)(\alpha, \alpha)}{\sin ^{2}(\alpha, x)}
$$


where $R_{+}$is a positive part of a root system $R$ (which could be non-reduced), and $m(\alpha)=m_{\alpha}$ is a function on $R$ which is invariant under the corresponding Weyl group $W$. If $R$ is a root system of a compact symmetric space $X$ and $m_{\alpha}=\frac{\mu_{\alpha}}{2}-1$ where $\mu_{\alpha}$ are the multiplicities of the roots then the operator (1) is conjugated to the radial part of the Laplace-Beltrami operator on $X$

$$
\mathcal{L}=-\Delta+2 \sum_{\alpha \in R_{+}} m_{\alpha} \cot (\alpha, x) \partial_{\alpha}
$$

namely

$$
\mathcal{L}=\hat{\psi}_{0}^{-1} \circ L \circ \hat{\psi}_{0}
$$

where $\hat{\psi}_{0}$ is the multiplication operator by the function

$$
\hat{\psi}_{0}=\prod_{\alpha \in R_{+}} \sin ^{-m_{\alpha}}(\alpha, x) .
$$

In this general form this was shown by Olshanetsky and Perelomov [4] but in the special case $X=S U(n) / S O(n)$ it was observed already in 1964 by Berezin et al in [5]. So integrability of the generalized CMS quantum problem (1) in this case follows from the general theory of the invariant operators on the symmetric spaces (see e.g. [6]). In the general case the proof of integrability was found later by Heckman and Opdam [7, 8] who used very different arguments.

It turned out however that there are other, non-symmetric integrable generalizations of the quantum CMS problems. The first series $A_{n}(m)$ of such "deformed" CMS problems have been found by Chalykh, Feigin and one of the authors in [9],[10], later another series $C_{n}(m, l)$ was discovered [11]. Although these deformations since then appeared in different context (see e.g. [12] about relations to WDVV equations) the algebraic nature of them until recently was totally unclear.

An important step was done by one of the authors in $[13,14]$ who observed that the deformed CMS operator of the type $A_{n}(m)$ for a special value of parameter can be interpreted as the radial part of Laplace-Beltrami operator on certain symmetric superspace. This indicated that the clue to this mystery could be found in this direction.

The goal of the present paper is to develop a systematic theory of the deformed CMS quantum systems related to Lie superalgebras and symmetric superspaces. A crucial role in our approach plays the notion of the generalized root systems introduced by V. Serganova in the paper [15].

Serganova suggested a version of the standard geometric description of the root systems [16] in the presence of the isotropic roots. She classified all such irreducible systems and showed that they are essentially the root systems of the contragredient Lie superalgebras classified by V. Kac in [17]. All the generalized root systems have partial symmetry described by the Weyl group $W_{0}$ generated by reflections corresponding to the non-isotropic ("real") roots. 
For any such generalized root system $R$ we construct a family of the deformed quantum CMS systems in the following way. The system $R$ stays the same, we change the scalar product and the multiplicities of the roots (which are not integers anymore) in such a way that the following conditions are satisfied:

1) the new bilinear form $B$ and the multiplicity function stay $W_{0}$-invariant

2) multiplicities of all the imaginary roots are fixed to be 1

3) the corresponding Schrödinger operator (1) has a radial form (2) or equivalently, has an eigenfunction of the form (3).

The last condition leads to certain relations between the multiplicities and parameters of the form. Our analysis shows that the admissible forms for all generalized root systems depend on one deformation parameter (in the case $D(2,1, \lambda)$ we have three parameters but two of them are already in the Lie superalbegra itself), although multiplicities have sometimes more freedom (see Section 2). We call the corresponding operators the deformed $C M S$ operators related to generalized root system $R$.

According to Serganova's classification we have two infinite series of such operators related to the classical systems $R$ of the type $A(n, m)$ and $B C(n, m)$, and three exceptional cases corresponding to the exceptional systems $G(1,2)$, $A B(1,3)$ and $D(2,1, \lambda)$. The deformed operators corresponding to the classical series have the form:

$$
\begin{aligned}
L_{A(n-1, m-1)}= & -\left(\frac{\partial^{2}}{\partial x_{1}{ }^{2}}+\cdots+\frac{\partial^{2}}{\partial x_{n}{ }^{2}}\right)-k\left(\frac{\partial^{2}}{\partial y_{1}{ }^{2}}+\cdots+\frac{\partial^{2}}{\partial y_{m}{ }^{2}}\right)+\sum_{i<j}^{n} \frac{2 k(k+1)}{\sin ^{2}\left(x_{i}-x_{j}\right)} \\
& +\sum_{i<j}^{m} \frac{2\left(k^{-1}+1\right)}{\sin ^{2}\left(y_{i}-y_{j}\right)}+\sum_{i=1}^{n} \sum_{j=1}^{m} \frac{2(k+1)}{\sin ^{2}\left(x_{i}-y_{j}\right)}
\end{aligned}
$$

for $A(n-1, m-1)$, where $k$ is an arbitrary parameter, and

$$
\begin{aligned}
L_{B C(n, m)}= & -\left(\frac{\partial^{2}}{\partial x_{1}{ }^{2}}+\cdots+\frac{\partial^{2}}{\partial x_{n}{ }^{2}}\right)-k\left(\frac{\partial^{2}}{\partial y_{1}{ }^{2}}+\cdots+\frac{\partial^{2}}{\partial y_{m}{ }^{2}}\right) \\
& +\sum_{i<j}^{n}\left(\frac{2 k(k+1)}{\sin ^{2}\left(x_{i}-x_{j}\right)}+\frac{2 k(k+1)}{\sin ^{2}\left(x_{i}+x_{j}\right)}\right)+\sum_{i<j}^{m}\left(\frac{2\left(k^{-1}+1\right)}{\sin ^{2}\left(y_{i}-y_{j}\right)}+\frac{2\left(k^{-1}+1\right)}{\sin ^{2}\left(y_{i}+y_{j}\right)}\right) \\
& +\sum_{i=1}^{n} \sum_{j=1}^{m}\left(\frac{2(k+1)}{\sin ^{2}\left(x_{i}-y_{j}\right)}+\frac{2(k+1)}{\sin ^{2}\left(x_{i}+y_{j}\right)}\right)+\sum_{i=1}^{n} \frac{p(p+2 q+1)}{\sin ^{2} x_{i}} \\
& +\sum_{i=1}^{n} \frac{4 q(q+1)}{\sin ^{2} 2 x_{i}}+\sum_{j=1}^{m} \frac{k r(r+2 s+1)}{\sin ^{2} y_{j}}+\sum_{j=1}^{m} \frac{4 k s(s+1)}{\sin ^{2} 2 y_{j}}
\end{aligned}
$$

for $B C(n, m)$, where the parameters $k, p, q, r, s$ satisfy the following relations

$$
p=k r, \quad 2 q+1=k(2 s+1)
$$

The system (4) can be considered as the interaction of two groups of particles of masses 1 and $\frac{1}{k}$ respectively with the special parameters of interaction 
depending on $k$. When $m=1$ ( i.e. when the second group consists only of one particle) this system was first proposed in [9]. For the general $n$ and $m$ the operator (4) was first introduced by one of the authors in [13] but its rational limit was discovered earlier by Berest and Yakimov, who were looking for a Darboux-type transformations for Calogero-Moser systems [18].

The system (5) can be interpreted in a similar way under the assumption that the configuration of particles is symmetric with respect to the origin. Although it depends on 5 parameters only 3 of them are independent due to relations (6) (say $k, p$ and $q$ ). The system $B C(n, m)$ with $m=1$ and $p=0$ was first considered in [11]. The case $m=1$ is special as the only one when all the parameters could be integer. As far as we know the operator (5) for the general $m, n$ as well as the deformed CMS systems related to the exceptional root systems $G(1,2), A B(1,3)$ and $D(2,1, \lambda)$ (see the next section) were not considered before.

The conjecture is that all these quantum systems are integrable in the sense that they have enough commuting integrals. In this paper we prove this conjecture for the classical series ( i.e. for the operators (4) and (5)) explicitly constructing the integrals.

The corresponding algebra of integrals seems to be interesting by itself. To describe it we introduce the following algebra $\Lambda_{R, B}$ related to a deformed generalized root system $(R, B)$. It consists of the polynomials $p(x)$ on $V$ which are invariant under reflections $s_{\alpha}$ corresponding to the real roots (i.e. are $W_{0}$-invariant) and satisfy the conditions

$$
p\left(x+\frac{1}{2} \alpha\right) \equiv p\left(x-\frac{1}{2} \alpha\right)
$$

on the hyperplane $B(\alpha, x)=0$ for each imaginary root $\alpha$.

Our main result can be formulated as follows.

Theorem. For the classical generalized root systems $R$ and generic values of the deformation parameter $k$ in the form $B$ there exists a monomorphism $\chi$ from the commutative algebra $\Lambda_{R, B}$ into the algebra of differential operators on $V$ such that $\chi\left(x^{2}\right)$ is the corresponding deformed CMS operator related to $R$.

In the rational limit a similar result holds for a closely related algebra $\Lambda_{R, B}^{0}$ with the condition (7) in the definition of $\Lambda_{R, B}$ replaced by its differential version: $\partial_{\alpha} p(x)=0$ when $B(\alpha, x)=0$.

The structure of the paper is following. We start with the precise definition of the generalized root systems $R$ and formulate Serganova's classification theorem. Then we define the deformed CMS operators related to such a system $R$ and classify all of them for each generalized root system.

In section 3 we prove the integrability of the deformed quantum CMS problems for the classical series $A(n, m)$ and $B C(n, m)$. The proof is effective: the quantum integrals are given explicitly by some recurrent formula. 
Our formula can be considered as a deformed version of the Matsuo's formula (2.3.6) from [19].

In section 4 we introduce and investigate the algebras $\Lambda_{R, B}$ and $\Lambda_{R, B}^{0}$. In particular we show that for the classical series and generic values of the deformation parameter $k$ these algebras are finitely generated and compute the Poincare series for them. We show that the image of the Harish-Chandra homomorphism from the rings of quantum integrals of the deformed CMS problems described in section 2 for generic $k$ is exactly the algebra $\Lambda_{R, B}$ (in

the rational case $-\Lambda_{R, B}^{0}$ ). In the last section 5 we discuss the elliptic and difference generalizations of our operators.

\section{Generalized Root Systems And Deformed quantum CMS PROBLEMS.}

We start with the definition of the generalized root systems due to $\mathrm{V}$. Serganova [15]. We should mention that there are three slightly different definitions of generalized root systems in [15], we choose one of them which suits best for our purpose.

Let $V$ be a finite dimensional complex vector space with a non-degenerate bilinear form $<,>$.

Definition. The finite set $R \subset V \backslash\{0\}$ is called a generalized root system if the following conditions are fulfilled :

1) $R$ spans $V$ and $R=-R$;

2) if $\alpha, \beta \in R$ and $\left\langle\alpha, \alpha>\neq 0\right.$ then $\frac{2<\alpha, \beta>}{<\alpha, \alpha>} \in \mathbf{Z}$ and $s_{\alpha}(\beta)=\beta-$ $\frac{2<\alpha, \beta>}{<\alpha, \alpha>} \alpha \in R$

3) if $\alpha \in R$ and $\langle\alpha, \alpha\rangle=0$ then for any $\beta \in R$ such that $\langle\alpha, \beta\rangle \neq 0$ at least one of the vectors $\beta+\alpha$ or $\beta-\alpha$ belongs to $R$.

The non-isotropic roots are called real, the isotropic roots are called $\mathrm{imag}$ inary:

$$
R_{r e}=\{\alpha \in R:<\alpha, \alpha>\neq 0\} \quad R_{i m}=\{\alpha \in R:<\alpha, \alpha>=0\}
$$

A generalized root system $R$ is called reducible if it can be represented as a direct sum of two non-empty generalized root systems $R_{1}$ and $R_{2}$, i.e. $V=V_{1} \oplus V_{2}, R_{1} \subset V_{1}, R_{2} \subset V_{2}$, and $R=R_{1} \cup R_{2}$. Otherwise the system is called irreducible.

Any generalized root system has a partial symmetry described by the finite group $W_{0}$ generated by the reflections with respect to the real roots.

The main result of a remarkable Serganova's paper [15] is the classification theorem for the irreducible generalized root systems which says that they all are contained in the following list.

\section{List of the irreducible generalized root systems.}

\section{Classical series}

1. $A(n-1, m-1), \quad n \neq m$ 
Let $V_{n, m}=V_{1} \oplus V_{2}$ be a vector space with the basis $\left\{e_{1}, \ldots, e_{n+m}\right\}$, such that $\left\{e_{1}, \ldots, e_{n}\right\}$ be a basis of $V_{1}$ and $\left\{e_{n+1}, \ldots, e_{n+m}\right\}$ be a basis of $V_{2}$. Let $e^{i}, i=1, \ldots, n+m$ denote the corresponding basis in the dual space $V_{n, m}^{*}$.

Consider the following bilinear (indefinite) symmetric form on $V_{n, m}$

$$
B(u, v)=\sum_{i=1}^{n} u^{i} v^{i}-\sum_{j=n+1}^{n+m} u^{j} v^{j},
$$

where $u^{i}, v^{i}$ are the coordinates of vectors $u, v$ in the basis $e_{i}$.

Let us split the set of the indices $I=\{1, \ldots, n+m\}$ into two groups: $I=I_{1} \cup I_{2}$, where $I_{1}=\{1, \ldots, n\}, I_{2}=\{n+1, \ldots, n+m\}$ and rewrite the last formula as

$$
B=\sum_{i \in I_{1}} e^{i} \otimes e^{i}-\sum_{j \in I_{2}} e^{j} \otimes e^{j}
$$

where $B$ is now considered as an element of $V^{*} \otimes V^{*}$.

The generalized root system of type $A(n-1, m-1), n \neq m$ is defined as the set $R=\left\{e_{i}-e_{j}, i \neq j, i, j \in I\right\}$ and the corresponding space $V$ is the hyperplane in $V_{n, m}$ generated by this set with the induced bilinear form. It is easy to see that in this case $R_{r e}=A_{n-1} \oplus A_{m-1}, R_{i m}=\left\{ \pm\left(e_{i}-e_{j}\right), i \in\right.$ $\left.I_{1}, j \in I_{2}\right\}$ Corresponding Lie superalgebra is $s l(n \mid m)$.

2. $A(n-1, n-1)$

If we would do the same when $m=n$ then we will have a problem: the restriction of the form $B$ on the corresponding hyperplane $V$ is degenerate. Indeed the vector $v=\sum_{i \in I_{1}} e_{i}-\sum_{j \in I_{2}} e_{j}$ belongs to $V$ and orthogonal to all the roots (and thus to all $V$ ). In order to have a proper generalized root system in this case we should consider the quotient $V^{\prime}=V /\langle v\rangle$ and the corresponding set $R^{\prime}$ which is the image of $R$ after such a projection. This is the system of the type $A(n-1, n-1)$. Corresponding Lie superalgebra is $\operatorname{psl}(n \mid n)$.

3. $B(n, m)$

In this case $V=V_{n, m}$ which is defined above with the same bilinear form $B$ and $R$ is the set $\left\{ \pm e_{i} \pm e_{j}, i \neq j, i, j \in I, \pm e_{i}, i \in I, \pm 2 e_{i}, i \in I_{2}\right\}$. The real and imaginary roots are $R_{r e}=B_{n} \oplus B C_{m}, R_{i m}=\left\{ \pm e_{i} \pm e_{j}, i \in I_{1}, j \in I_{2}\right\}$. This corresponds to the orthosymplectic Lie superalgebra $\operatorname{osp}(2 n+1 \mid 2 m)$.

4. $D(n, m), n \geq 2$

$V=V_{n, m}$ is the same as in the previous case, but $R$ is the set $\left\{ \pm e_{i} \pm e_{j}, i \neq\right.$ $\left.j, i, j \in I, \pm 2 e_{i}, i \in I_{2}\right\}$. We have $R_{r e}=D_{n} \oplus C_{m}, R_{i m}=\left\{ \pm e_{i} \pm e_{j}, i \in\right.$ $\left.I_{1}, j \in I_{2}\right\}$ Corresponding Lie superalgebra is $\operatorname{osp}(2 n \mid 2 m)$.

5. $C(0, m)$

Here $V=V_{1, m}$ and $R$ is the set $\left\{ \pm e_{i} \pm e_{j}, i \neq j, i, j \in I, \pm 2 e_{i}, i \in I_{2}\right\}$. In this case $R_{r e}=C_{m}, R_{i m}=\left\{ \pm e_{1} \pm e_{j}, j \in I_{2},\right\}$. Corresponding Lie superalgebra is $\operatorname{osp}(2 \mid 2 m)$.

6. $C(n, m)$

Here $V=V_{n, m}$ and $R$ is the set $\left\{ \pm e_{i} \pm e_{j}, i \neq j, i, j \in I, \pm 2 e_{i}, i \in I\right\}$, so that $R_{r e}=C_{n} \oplus C_{m}, R_{i m}=\left\{ \pm e_{i} \pm e_{j}, i \in I_{1}, j \in I_{2},\right\}$. In this case and in 
the next one there are no related Lie superalgebras but there are symmetric superspaces with such root systems.

7. $B C(n, m)$

$V=V_{n, m}$ and $R$ consists of $\left\{ \pm e_{i} \pm e_{j}, i \neq j, i, j \in I, \pm e_{i}, \pm 2 e_{i}, i \in I\right\}$. In this case $R_{r e}=B C_{n} \oplus B C_{m}, R_{i m}=\left\{ \pm e_{i} \pm e_{j}, i \in I_{1}, j \in I_{2}\right\}$

\section{Exceptional cases}

8. $A B(1,3)$ (also known as $F(4)$ )

Here $V=V_{1} \oplus V_{2}$, where $V_{1}$ is a three dimensional space with the basis $\left\{e_{1}, e_{2}, e_{3}\right\}$ and $V_{2}$ is a one-dimensional space generated by $e_{4}$. The bilinear form $B$ is

$$
B(u, v)=u^{1} v^{1}+u^{2} v^{2}+u^{3} v^{3}-3 u^{4} v^{4} .
$$

The root system $R$ is the set

$$
\pm e_{i} \pm e_{j}, \quad i \neq j, \quad \pm e_{i}, \quad i, j=1,2,3, \quad \pm e_{4}, \quad \frac{1}{2}\left( \pm e_{1} \pm e_{2} \pm e_{3} \pm e_{4}\right),
$$

$R_{r e}=B_{3} \oplus A_{1}, R_{i m}=\left\{\frac{1}{2}\left( \pm e_{1} \pm e_{2} \pm e_{3} \pm e_{4}\right)\right\}$

9. $G(1,2)$ (also known as $G(3)$ )

Here $V=V_{1} \oplus V_{2}$, where $V_{1}$ is a two-dimensional space, generated by three vectors $e_{1}, e_{2}, e_{3}$ with the condition that $e_{1}+e_{2}+e_{3}=0$ and $V_{2}$ is a one-dimensional space generated by $e_{4}$. The form $B$ is determined by the following conditions:

$B\left(e_{i}, e_{j}\right)=-1 \quad$ if $\quad i \neq j, \quad B\left(e_{i}, e_{i}\right)=2, \quad B\left(e_{i}, e_{4}\right)=0, \quad B\left(e_{4}, e_{4}\right)=-2$, where $i, j=1,2,3$.

$R$ consists of the vectors $\pm e_{i},\left(e_{i}-e_{j}\right), \pm e_{4}, \pm 2 e_{4}, \pm e_{i} \pm e_{4} \quad, i \neq j, i, j \leq 3$, $R_{r e}=G_{2} \oplus B C_{1}, R_{i m}=\left\{ \pm e_{i} \pm e_{4}, i=1,2,3\right\}$

10. $D(2,1, \lambda)$

Here $\lambda=\left(\lambda_{1}, \lambda_{2}, \lambda_{3}\right)$ are the parameters, satisfying the relation $\lambda_{1}+\lambda_{2}+$ $\lambda_{3}=0$.

The space $V$ is $V_{1} \oplus V_{2} \oplus V_{3}$ the direct sum of three one-dimensional spaces generated by $e_{1}, e_{2}, e_{3}$ respectively. The form $B$ is

$$
B(u, v)=\lambda_{1} u^{1} v^{1}+\lambda_{2} u^{2} v^{2}+\lambda_{3} u^{3} v^{3} .
$$

$R$ is the set $\left\{ \pm 2 e_{1}, \pm 2 e_{2}, \pm 2 e_{3}, \pm e_{1} \pm e_{2} \pm e_{3}\right\}, R_{r e}=A_{1} \oplus A_{1} \oplus A_{1}$, $R_{i m}=\left\{ \pm e_{1} \pm e_{2} \pm e_{3}\right\}$

Now we are going to explain how one can construct a family of the Schrödinger operators related to a generalized root system $R \subset V$.

These operators are defined on the same space $V$ and have the form

$$
L=-\Delta+\sum_{\alpha \in R_{+}} \frac{m_{\alpha}\left(m_{\alpha}+2 m_{2 \alpha}+1\right)(\alpha, \alpha)}{\sin ^{2}(\alpha, x)}
$$

but now the brackets $($,$) and the Laplacian \Delta$ correspond to the new ("deformed") bilinear form $B$ on $V$. Sometime we would consider this operator on $V^{*}$ (which can be identified with $V$ using $B$ ); in that case the brackets 
$(\alpha, x)$ should be understood as a natural pairing between vectors and covectors. The multiplicities $m_{\alpha}$ are related to $B$ in such a way that the following conditions are satisfied:

1) the new form $B$ and the multiplicities are $W_{0}$-invariant;

2) all imaginary roots have the multiplicity 1 ;

3) the function $\psi_{0}=\prod_{\alpha \in R_{+}} \sin ^{-m_{\alpha}}(\alpha, x)$ is a (formal) eigenfunction of the corresponding Schrödinger operator (10).

We will call such forms $B$ and multiplicities admissible and the corresponding operators (10) the deformed CMS operators related to generalized root system $R$. If we replace in (10) $x$ by $\omega x$ and let $\omega$ tend to 0 (in other words if we replace $\sin z$ by $z$ ) we will have the rational limits of these operators.

Let us comment on the conditions 1)-3). The first one is very natural: we would like to preserve the (partial) symmetry of the system. The third condition is responsible for the existence of the "radial gauge" of the operator $L$ :

$$
\mathcal{L}_{2}=-\Delta+2 \sum_{\alpha \in R^{+}} m_{\alpha} \cot (\alpha, x) \partial_{\alpha}
$$

(see [3], [20]), and thus is motivated by the theory of symmetric spaces. The second condition (related to condition 3) in Serganova's axiomatics of the generalized root systems) looks very simple but actually is the most difficult to justify. The motivation comes from the theory of the locus configurations, where the first examples of the such deformations have been found [10],[11]. In that theory all the multiplicities are integers and 1 is the smallest possible option.

A straightforward check shows that the condition 3) is equivalent to the following main identity:

$$
\sum_{\alpha \not \beta, \alpha, \beta \in R_{+}} m_{\alpha} m_{\beta}(\alpha, \beta)(\cot (\alpha, x) \cot (\beta, x)+1) \equiv 0,
$$

where $\alpha \nsim \beta$ means that $\alpha$ is not proportional to $\beta$ (note that in $B C(n, m)$ there are proportional roots). In that case $L \psi_{0}=\lambda \psi_{0}$ with the eigenvalue $\lambda=|\rho(m)|^{2}$ where $\rho(m)=\sum_{\alpha \in R_{+}} m_{\alpha} \alpha$ (cf. [3], [20]).

To describe all possible deformations for a given generalized root system one can use the fact that the condition (12) can be checked separately for all two-dimensional subsystems (cf. $[11,20]$ ). It is enough to consider only the following classical root systems $A(n, m), B C(n, m)$ (from deformation point of view others are simply special cases) and the exceptional root systems $G(1,2), A B(1,3), D(2,1, \lambda)$. The forms $B$ are obviously defined up to a multiple so we will choose some normalization to avoid unnecessary constants.

A straightforward analysis leads to the following

List of admissible deformations of generalized root systems. 
Here $B$ is considered as an element of $V^{*} \otimes V^{*}$ which is non-degenerate and thus determines an isomorphism between $V$ and its dual $V^{*}$. The formulas for the operators will be written on $V^{*}$ : this is more convenient for several reasons.

In all the cases the admissible forms depend on one parameter. We denote this parameter $k$ and choose it in such a way that the value $k=-1$ corresponds to the Lie superalgebra case.

\section{Classical series}

$A(n, m)$

Form $B$ is

$$
B=\sum_{i \in I_{1}} e^{i} \otimes e^{i}+k \sum_{j \in I_{2}} e^{j} \otimes e^{j},
$$

where $k$ is an arbitrary non-zero parameter. Multiplicities $m_{\alpha}=m(\alpha)$ of the real roots are

$$
m\left(e_{i}-e_{j}\right)=k, i, j \in I_{1}, \quad m\left(e_{i}-e_{j}\right)=k^{-1}, i, j \in I_{2}
$$

(recall that all imaginary roots have multiplicity 1 ).

Corresponding one-parameter family of the deformed CMS operators has the form (4). We should mention that in the case $m=n$ the vector $v=$ $\sum_{i \in I_{1}} e_{i}-\sum_{i \in I_{2}} e_{i}$ is not isotropic for the deformed form, so strictly speaking we deform not generalized system of type $A(n-1, n-1)$ but its (degenerate) extension.

$$
B C(n, m)
$$

$B$ is the same as above, multiplicities are

$$
\begin{gathered}
m\left(e_{i} \pm e_{j}\right)=k, \quad m\left(e_{i}\right)=p, \quad m\left(2 e_{i}\right)=q, i, j \in I_{1}, \\
m\left(e_{i} \pm e_{j}\right)=k^{-1}, \quad m\left(e_{j}\right)=r, \quad m\left(2 e_{j}\right)=s, i, j \in I_{2},
\end{gathered}
$$

where $p, q, r, s$ are satisfying the relations

$$
p=k r, \quad 2 q+1=k(2 s+1) .
$$

Corresponding deformed CMS operators depend on three free parameters and are given by (5).

\section{Exceptional cases}

$A B(1,3)$

$$
B=e^{1} \otimes e^{1}+e^{2} \otimes e^{2}+e^{3} \otimes e^{3}+3 k e^{4} \otimes e^{4},
$$

multiplicities are

$m\left(e_{i}\right)=a=\frac{3 k+1}{2}, \quad m\left(e_{4}\right)=b=\frac{1-k}{2 k}, \quad m\left(e_{i} \pm e_{j}\right)=c=\frac{3 k-1}{4}, i, j=1,2,3$.

Deformed CMS operator in this case has the form 


$$
\begin{aligned}
& L_{A B(1,3)}=-\left(\frac{\partial^{2}}{\partial x_{1}{ }^{2}}+\frac{\partial^{2}}{\partial x_{2}{ }^{2}}+\frac{\partial^{2}}{\partial x_{3}^{2}}\right)-3 k \frac{\partial^{2}}{\partial y^{2}}+\sum_{i=1}^{3} \frac{a(a+1)}{\sin ^{2} x_{i}}+\frac{3 k b(b+1)}{\sin ^{2} y} \\
& +\sum_{1 \leq i<j \leq 3}\left(\frac{4 c(c+1)}{\sin ^{2}\left(x_{i}-x_{j}\right)}+\frac{4 c(c+1)}{\sin ^{2}\left(x_{i}+x_{j}\right)}\right)+\frac{1}{4} \sum_{ \pm} \frac{(3 k+3)}{\sin ^{2} \frac{1}{2}\left(y \pm x_{1} \pm x_{2} \pm x_{3}\right)}
\end{aligned}
$$

where the parameters $a, b, c$ are given in terms of deformation parameter $k$ above and the last sum is over all 8 possible combinations of the signs. In this case we have only one free parameter $k$.

$G(1,2)$

In the basis $e_{1}, e_{2}, e_{4}$ the form $B$ has the form

$$
B=e^{1} \otimes e^{1}+e^{2} \otimes e^{2}-\frac{1}{2}\left(e^{1} \otimes e^{2}+e^{2} \otimes e^{1}\right)+k e^{4} \otimes e^{4} .
$$

Multiplicities are

$$
m\left(e_{i}\right)=a=1+2 k, \quad m\left(e_{i}-e_{j}\right)=b=\frac{2 k-1}{3}, \quad m\left(e_{4}\right)=c=\frac{1}{k}+2, \quad m\left(2 e_{4}\right)=d=\frac{1}{2 k}-\frac{1}{2},
$$

where $i, j=1,2,3$.

Corresponding deformation of CMS operator has the form

$$
\begin{aligned}
L_{G(1,2)=} & -\left(\frac{\partial^{2}}{\partial x_{1}{ }^{2}}-\frac{\partial^{2}}{\partial x_{1} \partial x_{2}}+\frac{\partial^{2}}{\partial x_{2}{ }^{2}}\right)-k \frac{\partial^{2}}{\partial y^{2}}+\sum_{i=1}^{3} \frac{a(a+1)}{\sin ^{2} x_{i}}+\sum_{1 \leq i<j \leq 3} \frac{3 b(b+1)}{\sin ^{2}\left(x_{i}-x_{j}\right)} \\
& +\frac{k c(c+2 d+1)}{\sin ^{2} y}+\frac{4 k d(d+1)}{\sin ^{2} 2 y}+\sum_{i=1}^{3}\left(\frac{2(k+1)}{\sin ^{2}\left(x_{i}-y\right)}+\frac{2(k+1)}{\sin ^{2}\left(x_{i}+y\right)}\right)
\end{aligned}
$$

where the parameters $a, b, c, d$ are given in terms of $k$ above. Again we have one-parameter family.

$$
D(2,1, \lambda)
$$

The form $B$ is

$$
B=\lambda_{1} e^{1} \otimes e^{1}+\lambda_{2} e^{2} \otimes e^{2}+\lambda_{3} e^{3} \otimes e^{3},
$$

where $\lambda_{i}, \quad i=1,2,3$ are arbitrary non-zero parameters. Let us introduce the parameter

$$
k=\lambda_{1}+\lambda_{2}+\lambda_{3}-1
$$

so that when $k=-1$ we have the Lie superalgebra case. The multiplicities have the form

$$
m\left(2 e_{i}\right)=m_{i}=\frac{k+1}{2 \lambda_{i}}-1, \quad i=1,2,3 .
$$

Corresponding deformed CMS operators are

$$
L_{D(2,1, \lambda)}=\lambda_{1} \frac{\partial^{2}}{\partial x_{1}{ }^{2}}+\lambda_{2} \frac{\partial^{2}}{\partial x_{2}^{2}}+\lambda_{3} \frac{\partial^{2}}{\partial x_{3}{ }^{2}}+\sum_{i=1}^{3} \frac{4 \lambda_{i} m_{i}\left(m_{i}+1\right)}{\sin ^{2} 2 x_{i}}+\sum_{ \pm} \frac{2(k+1)}{\sin ^{2}\left(x_{1} \pm x_{2} \pm x_{3}\right)}
$$


where the last sum is again over all possible combinations of signs (4 in this case). This family is living on the projective plane with projective coordinates $\lambda_{1}: \lambda_{2}: \lambda_{3}$. The Lie superalgebra case corresponds to the line $\lambda_{1}+\lambda_{2}+\lambda_{3}=0$, so we have again only one deformation parameter (say $k$ ).

It is interesting to note that the potentials $U$ of all operators listed above satisfy the so-called locus conditions (see [11]): the first coefficient in the Laurent expansion of $U$ in the direction of $\alpha$ is identically zero on the hyperplanes $\sin (\alpha, x)=0$. For the real roots this is obvious by symmetry reasons, but for imaginary roots this is not and follows only from a direct case by case check. If we replace the third condition for admissible deformations by these locus relations we will have essentially the same list of the potentials (some additional possibilities are due simply to the symmetry of the potential under the change $\left.m_{\alpha} \rightarrow\left(-1-m_{\alpha}\right)\right)$. We do not have satisfactory explanation of this phenomenon, which seems to be important (cf. $[20],[21])$.

Since the locus configurations (in the rational case) are known to be related to the Huygens' Principle (see [11]) it is natural to ask about the deformed CMS operators with all multiplicities being integers. A simple check of the cases listed above shows that the only non-trivial cases correspond to the systems $A(n, 1)$ and $B C(n, 1)$ which has already been discovered in $[10],[11]$. This indicates that the list of known locus configurations [21] could be in fact complete and therefore could give the answer to the famous Hadamard problem in the theory of Huygens' principle.

\section{Construction of Quantum integrals For Classical SERIES.}

In this section we present a recursive formula for the quantum integrals of the deformed CMS problems (4),(5) (more precisely, for its hyperbolic versions). Our formula is a properly deformed version of the formula used by A. Matsuo in his paper [19] on the relations between CMS quantum problem and $\mathrm{KZ}$ equation. We would like to mention also that for $m=1$ an equivalent set of integrals was found earlier in [10] using very different ideas.

Let $V$ be $n+m$-dimensional vector space with the deformed inner product

$$
(u, v)=\sum_{i=1}^{n} u^{i} v^{i}+k \sum_{j=n+1}^{n+m} u^{j} v^{j}
$$

corresponding to the form $B$ given by (13).

When $k=1$ we have the standard Euclidean scalar product which we denote by $<,>$ :

$$
<u, v>=u^{1} v^{1}+\ldots+u^{n+m} v^{n+m} .
$$

We will be using the fact that the generalized root systems $R$ of type $A(n-$ $1, m-1)$ and $B C(n, m)$ with respect to the scalar product $\langle u, v\rangle$ coincide with the usual root systems $A(n+m-1)$ and $B C(n+m)$ (see previous 
section). The corresponding Weyl groups $W$ are generated by reflections with respect to the Euclidean form which we denote as $s_{\langle\alpha\rangle}$.

Let us introduce the operator $\mathcal{B}$ by the relation

$$
B(u, v)=<\mathcal{B} u, v>\text { : }
$$

$\mathcal{B} e_{i}=e_{i}, i=1, \ldots, n, \quad \mathcal{B} e_{j}=k e_{j}, j=n+1, \ldots, n+m$. We will call a vector $v \in V$ homogeneous if it is an eigenvector of $\mathcal{B}$ (and thus for any pair of forms in our family).

For any homogeneous vector $v$ the following relation holds:

$$
\frac{(x, v)}{<x, v>}=\frac{(v, v)}{<v, v>}
$$

for any vector $x \in V$. Obviously in our case the set of homogeneous vectors is $V_{1} \cup V_{2}$, where $V_{1}$ and $V_{2}$ are generated by the first $n$ and last $m$ basic vectors respectively. It will be important for us that for the classical systems there exists an orbit $\mathcal{O}$ of the Weyl group $W$, which consists of homogeneous vectors. Indeed, for $A(n-1, m-1)$ root system one can take $\mathcal{O}=e_{i}$ and for $B C(n, m)$ such an orbit is $\mathcal{O}= \pm e_{i}$.

In this section we will assume that $x \in V$ and the brackets $(\alpha, x)$ denote the deformed product given by $B$.

Let us define for $\alpha \in R$ the following functions on $V$

$$
f_{\alpha}(x)=\frac{1}{2} \frac{e^{(\alpha, x)}+1}{e^{(\alpha, x)}-1}=\frac{1}{2} \operatorname{coth} \frac{(\alpha, x)}{2}, \quad \varphi_{\alpha}(x)=\frac{1}{4}-f_{\alpha}(x)^{2}=-\frac{1}{4 \sinh ^{2} \frac{(\alpha, x)}{2}} .
$$

They satisfy the following relations:

$$
\partial_{v} f_{\alpha}=(v, \alpha) \varphi_{\alpha}, \quad \partial_{v} \varphi_{\alpha}=-2(v, \alpha) f_{\alpha} \varphi_{\alpha}
$$

for any $v \in V$.

Let us define now for any natural number $p$ the operator $\partial_{v}^{(p)}$ by the following recurrent procedure

$$
\begin{gathered}
\partial_{v}^{(1)}=\partial_{v}, \\
\partial_{v}^{(p)}=\partial_{v} \partial_{v}^{(p-1)}-\sum_{\alpha \in R^{+}} m_{\alpha}(\alpha, v) f_{\alpha}\left(\partial_{v}^{(p-1)}-\partial_{s<\alpha>v}^{(p-1)}\right),
\end{gathered}
$$

where $R^{+}$is a positive part of $R$ and $s_{<\alpha>}$ is the reflection corresponding to the root $\alpha$ with respect to the Euclidean form $<,>$. The formula (17) is a deformed version of the formula (2.3.6) from A. Matsuo's paper [19].

Take now an orbit $\mathcal{O}$ of the corresponding Weyl group $W$ consisting of homogeneous elements (see above) and define

$$
\mathcal{L}_{p}=\sum_{v \in \mathcal{O}} \frac{\partial_{v}^{(p)}}{(v, v)}
$$

One can easily check using the relation

$$
\partial_{v}-\partial_{s<\alpha>v}=2 \frac{<\alpha, v>}{<\alpha, \alpha>} \partial_{\alpha}
$$


that

$$
\mathcal{L}_{2}=\sum_{v \in \mathcal{O}} \frac{\partial_{v}^{2}}{(v, v)}-2 \sum_{\alpha \in R^{+}} m_{\alpha} f_{\alpha} \partial_{\alpha}
$$

is up to a coefficient the deformed CMS operator (11) (in the hyperbolic version and radial gauge) after a scaling $x \rightarrow 2 x$.

Theorem 1. The operators $\mathcal{L}_{p}$ given by the formula (18) commute with each other:

$$
\left[\mathcal{L}_{p}, \mathcal{L}_{q}\right]=0
$$

and thus are the quantum integrals of the corresponding deformed CMS problem (11) related to classical generalized root systems.

The proof is based on the following

Proposition 1. The operators $\partial_{v}^{(p)}$ satisfy the following commutation relation with the deformed CMS operator:

$$
\left[\mathcal{L}_{2}, \partial_{v}^{(p)}\right]=(v, v) \sum_{\alpha \in R^{+}} m_{\alpha} \frac{<\alpha, \alpha>}{<v, v>} \varphi_{\alpha}\left(\partial_{v}^{(p)}-\partial_{s_{<\alpha>v}}^{(p)}\right)
$$

The proof is by induction in $p$. For $p=1$ we have the relation

$$
\left[\mathcal{L}_{2}, \partial_{v}\right]=(v, v) \sum_{\alpha \in R^{+}} m_{\alpha} \frac{\langle\alpha, \alpha>}{<v, v>} \varphi_{\alpha}\left(\partial_{v}-\partial_{s<\alpha>v}\right),
$$

which is easy to check.

The proof of the induction step is a long but straightforward calculation. We reproduce the main steps to show the role of the properties of admissible deformations here.

Let us assume that the statement is true for all natural numbers less than $p$. We have

$$
\begin{aligned}
& {\left[\mathcal{L}_{2}, \partial_{v}^{(p)}\right]=\left[\mathcal{L}_{2}, \partial_{v}\right] \partial_{v}^{(p-1)}+\partial_{v}\left[\mathcal{L}_{2}, \partial_{v}^{(p-1)}\right]-\sum_{\alpha \in R^{+}} m_{\alpha}(\alpha, v)\left[\mathcal{L}_{2}, f_{\alpha}\right]\left(\partial_{v}^{(p-1)}-\partial_{s<\alpha>v}^{(p-1)}\right)} \\
& -\sum_{\alpha \in R^{+}} m_{\alpha}(\alpha, v) f_{\alpha}\left[\mathcal{L}_{2}, \partial_{v}^{(p-1)}-\partial_{s_{<\alpha}>v}^{(p-1)}\right]=(v, v) \sum_{\alpha \in R^{+}} m_{\alpha} \frac{<\alpha, \alpha>}{<v, v>} \varphi_{\alpha}\left(\partial_{v}-\partial_{s_{<\alpha>v}}\right) \partial_{v}^{(p-1)} \\
& +(v, v) \partial_{v} \sum_{\alpha \in R^{+}} m_{\alpha} \frac{<\alpha, \alpha>}{<v, v>} \varphi_{\alpha}\left(\partial_{v}^{(p-1)}-\partial_{s<\alpha>v}^{(p-1)}\right)-2 \sum_{\alpha \in R^{+}} m_{\alpha}(\alpha, v) \varphi_{\alpha} \partial_{\alpha}\left(\partial_{v}^{(p-1)}-\partial_{s_{<\alpha}>v}^{(p-1)}\right) \\
& +2 \sum_{\alpha \in R^{+}} m_{\alpha}(\alpha, v) f_{\alpha} \partial_{\alpha}\left(f_{\alpha}\right)\left(\partial_{v}^{(p-1)}-\partial_{s_{<\alpha>v}}^{(p-1)}\right)-\sum_{\alpha \in R^{+}} m_{\alpha}(\alpha, v) f_{\alpha}\left[\mathcal{L}_{2}, \partial_{v}^{(p-1)}-\partial_{s_{<\alpha>v}}^{(p-1)}\right] \\
& +2 \sum_{\alpha, \beta \in R^{+}} m_{\alpha} m_{\beta}(\alpha, v) f_{\beta} \partial_{\beta}\left(f_{\alpha}\right)\left(\partial_{v}^{(p-1)}-\partial_{s_{<\alpha>v}}^{(p-1)}\right)
\end{aligned}
$$

where we have used the induction assumption and the relation

$$
\left[\mathcal{L}_{2}, f_{\alpha}\right]=2 \varphi_{\alpha} \partial_{\alpha}-2 f_{\alpha} \partial_{\alpha}\left(f_{\alpha}\right)-2 \sum_{\beta \in R^{+}} m_{\beta} f_{\beta} \partial_{\beta}\left(f_{\alpha}\right)
$$


Let us denote the sum of the last two sums in the previous expression as $B$ :

$B=2 \sum_{\alpha, \beta \in R^{+}} m_{\alpha} m_{\beta}(\alpha, v) f_{\beta} \partial_{\beta}\left(f_{\alpha}\right)\left(\partial_{v}^{(p-1)}-\partial_{s_{<\alpha>v}}^{(p-1)}\right)-\sum_{\alpha \in R^{+}} m_{\alpha}(\alpha, v) f_{\alpha}\left[\mathcal{L}_{2}, \partial_{v}^{(p-1)}-\partial_{s_{<\alpha>v}}^{(p-1)}\right]$

and the rest of the previous expression as $A$. Using the homogeneity of $v$ we can rewrite $A$ as

$$
\begin{aligned}
& A=(v, v) \sum_{\alpha \in R^{+}} m_{\alpha} \frac{<\alpha, \alpha>}{<v, v>} \varphi_{\alpha}\left\{\left(\partial_{v}-\partial_{s<\alpha>v}\right) \partial_{v}^{(p-1)}+\partial_{v}\left(\partial_{v}^{(p-1)}-\partial_{s<\alpha>v}^{(p-1)}\right)\right\} \\
& +(v, v) \sum_{\alpha \in R^{+}} m_{\alpha} \frac{<\alpha, \alpha>}{<v, v>} \partial_{v}\left(\varphi_{\alpha}\right)\left(\partial_{v}^{(p-1)}-\partial_{s_{<\alpha>v}}^{(p-1)}\right)-2 \sum_{\alpha \in R^{+}} m_{\alpha}(\alpha, v) \varphi_{\alpha} \partial_{\alpha}\left(\partial_{v}^{(p-1)}-\partial_{s_{<\alpha>v}}^{(p-1)}\right) \\
& +2 \sum_{\alpha \in R^{+}} m_{\alpha}(\alpha, v) f_{\alpha} \partial_{\alpha}\left(f_{\alpha}\right)\left(\partial_{v}^{(p-1)}-\partial_{s<\alpha>v}^{(p-1)}\right) \\
& =(v, v) \sum_{\alpha \in R^{+}} m_{\alpha} \frac{<\alpha, \alpha>}{<v, v>} \varphi_{\alpha}\left(\partial_{v} \partial_{v}^{(p-1)}-\partial_{s_{\alpha} v} \partial_{s_{<\alpha>v}}^{(p-1)}\right) \\
& +2 \sum_{\alpha \in R^{+}} m_{\alpha}(\alpha, v) f_{\alpha}\left(\partial_{\alpha}\left(f_{\alpha}\right)-\frac{(v, v)}{<v, v>}<\alpha, \alpha>\varphi_{\alpha}\right)\left(\partial_{v}^{(p-1)}-\partial_{s<\alpha>v}^{(p-1)}\right) .
\end{aligned}
$$

Now let us use that

$$
\begin{gathered}
\partial_{v} \partial_{v}^{(p-1)}=\partial_{v}^{(p)}+\sum_{\beta \in R^{+}} m_{\beta}(\beta, v) f_{\beta}\left(\partial_{v}^{(p-1)}-\partial_{s_{<\beta>v}}^{(p-1)}\right) \\
\partial_{s_{<\alpha>v}} \partial_{s_{<\alpha>v}}^{(p-1)}=\partial_{s_{<\alpha>v}}^{(p)}+\sum_{\beta \in R^{+}} m_{\beta}\left(\beta, s_{<\alpha>v}\right) f_{\beta}\left(\partial_{s_{<\alpha>v}}^{(p-1)}-\partial_{s_{<\beta>s<\alpha>v}}^{(p-1)}\right),
\end{gathered}
$$

to rewrite the last expression as

$$
\begin{aligned}
& A=(v, v) \sum_{\alpha \in R^{+}} m_{\alpha} \frac{<\alpha, \alpha>}{<v, v>} \varphi_{\alpha}\left(\partial_{v}^{(p)}-\partial_{s_{<\alpha>v}}^{(p)}\right) \\
& +(v, v) \sum_{\alpha \in R^{+}} m_{\alpha} \frac{\langle\alpha, \alpha>}{<v, v>} \varphi_{\alpha} \sum_{\beta \in R^{+}} m_{\beta}(\beta, v) f_{\beta}\left(\partial_{v}^{(p-1)}-\partial_{s_{<\beta>}}^{(p-1)}\right) \\
& -(v, v) \sum_{\alpha \in R^{+}} m_{\alpha} \frac{<\alpha, \alpha>}{<v, v>} \varphi_{\alpha} \sum_{\beta \in R^{+}} m_{\beta}(\beta, s<\alpha>v) f_{\beta}\left(\partial_{s_{<\alpha>v}}^{(p-1)}-\partial_{s_{<\beta>}>\alpha>v}^{(p-1)}\right) \\
& +2 \sum_{\alpha \in R^{+}} m_{\alpha}(\alpha, v) f_{\alpha}\left(\partial_{\alpha}\left(f_{\alpha}\right)-\frac{(v, v)}{<v, v>}<\alpha, \alpha>\varphi_{\alpha}\right)\left(\partial_{v}^{(p-1)}-\partial_{s<\alpha>v}^{(p-1)}\right) \\
& =(v, v) \sum_{\alpha \in R^{+}} m_{\alpha} \frac{<\alpha, \alpha>}{<v, v>} \varphi_{\alpha}\left(\partial_{v}^{(p)}-\partial_{s<\alpha>v}^{(p)}\right) \\
& +(v, v) \sum_{\alpha \in R^{+}} m_{\alpha} \frac{<\alpha, \alpha>}{<v, v>} \varphi_{\alpha} m_{\alpha} f_{\alpha}\left((\alpha, v)+\left(\alpha, s_{<\alpha>v)}\right)\left(\partial_{v}^{(p-1)}-\partial_{s_{<\alpha>v}}^{(p-1)}\right)\right. \\
& +2 \sum_{\alpha \in R^{+}} m_{\alpha}(\alpha, v) f_{\alpha} \varphi_{\alpha}\left((\alpha, \alpha)-\frac{(v, v)}{<v, v>}<\alpha, \alpha>\right)\left(\partial_{v}^{(p-1)}-\partial_{s<\alpha>v}^{(p-1)}\right)
\end{aligned}
$$




$$
\begin{gathered}
+(v, v) \sum_{\alpha \in R^{+}} m_{\alpha} \frac{\langle\alpha, \alpha>}{<v, v>} \varphi_{\alpha} \sum_{\beta \in R^{+}, \beta \neq \alpha} m_{\beta}(\beta, v) f_{\beta}\left(\partial_{v}^{(p-1)}-\partial_{s_{<\beta>v}}^{(p-1)}\right) \\
-(v, v) \sum_{\alpha \in R^{+}} m_{\alpha} \frac{<\alpha, \alpha>}{<v, v>} \varphi_{\alpha} \sum_{\beta \in R^{+}, \beta \neq \alpha} m_{\beta}\left(\beta, s_{<\alpha>}>\right) f_{\beta}\left(\partial_{s<\alpha>v}^{(p-1)}-\partial_{s_{<\beta>s<\alpha>v}}^{(p-1)}\right) .
\end{gathered}
$$

Combining the second and third sums we come to the following expression

$$
\left(m_{\alpha}\left\{(\alpha, v)+\left(\alpha, s_{<\alpha>v)}\right\}+2<\alpha, v>\left\{\frac{(\alpha, \alpha)}{<\alpha, \alpha>}-\frac{(v, v)}{<v, v>}\right\}\right),\right.
$$

which can be rewritten in the form

$$
-2<\alpha, v>\left(m_{\alpha}-1\right)\left(\frac{(\alpha, \alpha)}{<\alpha, \alpha>}-\frac{(v, v)}{<v, v>}\right) .
$$

We claim that this is 0 for any root $\alpha$. Indeed if $\alpha$ is imaginary then $m_{\alpha}=1$ by our assumption (property 2 of admissible deformations). If $\alpha$ is real and $\left\langle\alpha, v>\right.$ is not zero, then $\left(\frac{(\alpha, \alpha)}{<\alpha, \alpha\rangle}-\frac{(v, v)}{<v, v\rangle}\right)=0$ for any $v$ from our orbit.

Thus we come to the following expression for $A$ :

$$
\begin{gathered}
A=(v, v) \sum_{\alpha \in R^{+}} m_{\alpha} \frac{\langle\alpha, \alpha>}{<v, v>} \varphi_{\alpha}\left(\partial_{v}^{(p)}-\partial_{s_{<\alpha>v}}^{(p)}\right) \\
\left.+(v, v) \sum_{\alpha \in R^{+}} m_{\alpha} \frac{<\alpha, \alpha>}{<v, v>} \varphi_{\alpha} \sum_{\beta \in R^{+} \beta \neq \alpha} m_{\beta}(\beta, v) f_{\beta} \partial_{v}^{(p-1)}-\partial_{s_{<\beta>v}}^{(p-1)}\right) \\
-(v, v) \sum_{\alpha \in R^{+}} m_{\alpha} \frac{<\alpha, \alpha>}{<v, v>} \varphi_{\alpha} \sum_{\beta \in R^{+} \beta \neq \alpha} m_{\beta}\left(\beta, s_{<\alpha>} v\right) f_{\beta}\left(\partial_{s<\alpha>v}^{(p-1)}-\partial_{s_{<\beta>}>\alpha>v}^{(p-1)}\right) .
\end{gathered}
$$

Now let us look at term $B$. We have

$$
\begin{aligned}
& \sum_{\alpha \in R^{+}} m_{\alpha}(\alpha, v) f_{\alpha}\left[\mathcal{L}_{2}, \partial_{v}^{(p-1)}-\partial_{s<\alpha>v}^{(p-1)}\right] \\
& =\sum_{\alpha, \beta \in R^{+}} m_{\alpha}(\alpha, v) f_{\alpha} m_{\beta}<\beta, \beta>\varphi_{\beta} \frac{(v, v)}{<v, v>}\left(\partial_{v}^{(p-1)}-\partial_{s_{<\beta>v}}^{(p-1)}\right) \\
& -\sum_{\alpha, \beta \in R^{+}} m_{\alpha}(\alpha, v) f_{\alpha} m_{\beta}<\beta, \beta>\varphi_{\beta} \frac{\left(s_{<\alpha>} v, s_{<\alpha>} v\right)}{<s_{<\alpha>} v, s_{<\alpha>}>}\left(\partial_{s_{<\alpha>v}}^{(p-1)}-\partial_{s_{<\beta>s<\alpha>}}^{(p-1)}\right) \\
& =\sum_{\alpha, \beta \in R^{+} \beta \neq \alpha} m_{\alpha}(\alpha, v) f_{\alpha} m_{\beta}<\beta, \beta>\varphi_{\beta} \frac{(v, v)}{<v, v>}\left(\partial_{v}^{(p-1)}-\partial_{s_{<\beta>v}}^{(p-1)}\right) \\
& -\sum_{\alpha, \beta \in R^{+} \beta \neq \alpha} m_{\alpha}(\alpha, v) f_{\alpha} m_{\beta}<\beta, \beta>\varphi_{\beta} \frac{\left(s_{<\alpha>} v, s_{<\alpha>} v\right)}{<s_{<\alpha>} v, s_{<\alpha>} v>}\left(\partial_{s_{<\alpha>}}^{(p-1)}-\partial_{s_{<\beta>}<\alpha>v}^{(p-1)}\right) \\
& +\sum_{\alpha \in R^{+}} m_{\alpha}^{2}(\alpha, v) f_{\alpha}<\alpha, \alpha>\varphi_{\alpha}\left(\frac{(v, v)}{\langle v, v>}+\frac{\left(s_{<\alpha>} v, s_{<\alpha>} v\right)}{\left\langle s_{<\alpha>}>, s_{<\alpha>}>\right.}\right)\left(\partial_{v}^{(p-1)}-\partial_{s<\alpha>v}^{(p-1)}\right) .
\end{aligned}
$$


Combining the last term with the term in the second sum of $B$ corresponding to $\beta=\alpha$ and using the relation

$$
\left(s_{<\alpha>} v, s_{<\alpha>} v\right)=(v, v)+4 \frac{<\alpha, v>^{2}}{<\alpha, \alpha>}\left(\frac{(\alpha, \alpha)}{<\alpha, \alpha>}-\frac{(v, v)}{<v, v>}\right)
$$

we have

$m_{\alpha}^{2}(\alpha, v) f_{\alpha}\left\{2 \partial_{\alpha}\left(f_{\alpha}\right)-<\alpha, \alpha>\varphi_{\alpha}\left(\frac{(v, v)}{<v, v>}+\frac{\left(s_{<\alpha>} v, s_{<\alpha>} v\right)}{<s_{<\alpha>} v, s_{<\alpha>} v>}\right)\right\}\left(\partial_{v}^{(p-1)}-\partial_{s_{<\alpha>v}}^{(p-1)}\right)$,

which is zero since

$$
2\left(1-2 \frac{<\alpha, v>^{2}}{<\alpha, \alpha><v, v>}\right)\left(\frac{(\alpha, \alpha)}{<\alpha, \alpha>}-\frac{(v, v)}{<v, v>}\right)\left(\partial_{v}^{(p-1)}-\partial_{s_{<\alpha>v}}^{(p-1)}\right)=0
$$

for any root: for the real roots the product of the last two brackets is zero while for imaginary roots the first bracket is zero.

Thus we arrive at the following expression for

$$
\begin{gathered}
{\left[\mathcal{L}_{2}, \partial_{v}^{(p)}\right]=A+B=(v, v) \sum_{\alpha \in R^{+}} m_{\alpha} \frac{<\alpha, \alpha>}{<v, v>} \varphi_{\alpha}\left(\partial_{v}^{(p)}-\partial_{s_{<\alpha>v}}^{(p)}\right)} \\
+(v, v) \sum_{\beta \neq \alpha} m_{\alpha} m_{\beta} \frac{<\alpha, \alpha>}{<v, v>} \varphi_{\alpha} f_{\beta}\left\{(\beta, v)\left(\partial_{v}^{(p-1)}-\partial_{s_{<\beta>v}}^{(p-1)}\right)-\left(\beta, s_{<\alpha>v}\right)\left(\partial_{s_{<\alpha}>v}^{(p-1)}-\partial_{s_{<\beta>s<\alpha}>v}^{(p-1)}\right)\right\}- \\
\sum_{\beta \neq \alpha} m_{\alpha} m_{\beta}(\alpha, v) \frac{<\beta, \beta>}{<v, v>} f_{\alpha} \varphi_{\beta}\left\{(v, v)\left(\partial_{v}^{(p-1)}-\partial_{s_{<\beta>v}}^{(p-1)}\right)-\left(s_{<\alpha>v}, s_{<\alpha>v}\right)\left(\partial_{s_{<\alpha>v}}^{(p-1)}-\partial_{s_{<\beta>s<\alpha}>v}^{(p-1)}\right)\right\} \\
+2 \sum_{\alpha \neq \beta} m_{\alpha} m_{\beta}(\alpha, v) f_{\beta} \partial_{\beta}\left(f_{\alpha}\right)\left(\partial_{v}^{(p-1)}-\partial_{s_{<\alpha>v}}^{(p-1)}\right) .
\end{gathered}
$$

Let us denote the sum of the last three sums in the previous expression as $C$. We must show that $C$ is identically zero. Let us notice that

$$
\sum_{\alpha \neq \beta} m_{\alpha} m_{\beta}(\alpha, v) f_{\beta} \partial_{\beta}\left(f_{\alpha}\right)=\sum_{\alpha \neq \beta} m_{\alpha} m_{\beta}(\alpha, v) f_{\beta}(\beta, \alpha) \varphi_{\alpha}=\partial_{v}\left(\sum_{\alpha \neq \beta} m_{\alpha} m_{\beta}(\alpha, \beta) f_{\beta} f_{\alpha}\right)
$$

But according to our assumption (third property of admissible deformations)

$$
\sum_{\alpha \neq \beta} m_{\alpha} m_{\beta}(\alpha, \beta) f_{\beta} f_{\alpha}=\text { const }+4 \sum_{\alpha} m_{\alpha} m_{2 \alpha}(\alpha, \alpha) f_{2 \alpha} f_{\alpha}
$$

(see the formula (12) above). Thus $C$ can be rewritten as

$$
\begin{aligned}
& \sum_{\beta \neq \alpha}(v, v) m_{\alpha} m_{\beta} \frac{<\alpha, \alpha>}{<v, v>} \varphi_{\alpha} f_{\beta}\left\{(\beta, v)\left(\partial_{v}^{(p-1)}-\partial_{s_{<\beta>v}}^{(p-1)}\right)-\left(\beta, s_{<\alpha>v}\right)\left(\partial_{s_{<\alpha}>v}^{(p-1)}-\partial_{s_{<\beta>s}<\alpha>v}^{(p-1)}\right)\right\} \\
& -\sum_{\beta \neq \alpha} m_{\alpha} m_{\beta}(\alpha, v) \frac{<\beta, \beta>}{<v, v>} f_{\alpha} \varphi_{\beta}\left\{(v, v)\left(\partial_{v}^{(p-1)}-\partial_{s_{<\beta}>v}^{(p-1)}\right)-\left(s_{<\alpha>} v, s_{<\alpha>v}\right)\left(\partial_{s_{<\alpha}>v}^{(p-1)}-\partial_{s_{<\beta}>s<\alpha>v}^{(p-1)}\right)\right\} \\
& \quad-2 \sum_{\alpha \neq \beta} m_{\alpha} m_{\beta}(\alpha, v) f_{\beta} \partial_{\beta}\left(f_{\alpha}\right) \partial_{s_{<\alpha>v}}^{(p-1)}+4 \partial_{v}\left(\sum_{\alpha} m_{\alpha} m_{2 \alpha}(\alpha, \alpha) f_{2 \alpha} f_{\alpha}\right) \partial_{v}^{(p-1)}
\end{aligned}
$$




$$
=\sum_{\alpha \neq \beta} S_{1}(\alpha, \beta, v)-\sum_{\alpha \neq \beta} S_{2}(\alpha, \beta, v)-\sum_{\alpha \neq \beta} S_{3}(\alpha, \beta, v)+\sum_{\alpha} S_{4}(\alpha, v),
$$

where $S_{1}, S_{2}, S_{3}, S_{4}$ denote the terms in the first, second, third and forth sums respectively. Choose in the first three sums the terms with $\beta=2 \alpha$. We have $\sum_{\alpha} S_{1}(\alpha, 2 \alpha, v)=\sum_{\alpha} S_{1}(2 \alpha, \alpha, v)=0$ and

$-\sum_{\alpha}\left(S_{2}(\alpha, 2 \alpha, v)+S_{2}(2 \alpha, \alpha, v)\right)-\sum_{\alpha}\left(S_{3}(\alpha, 2 \alpha, v)+S_{3}(2 \alpha, \alpha, v)\right)+\sum_{\alpha} S_{4}(\alpha, v)=0$.

Thus we must show only that

$$
C=\sum_{\alpha \nsim \beta, \alpha, \beta \in R_{+}}\left(S_{1}(\alpha, \beta, v)-S_{2}(\alpha, \beta, v)-S_{3}(\alpha, \beta, v)\right)=0 .
$$

This can be done separately for each two-dimensional subsystems (cf. [11]). Proposition 1 is proven.

Now we are ready to prove Theorem 1 . When $p=2$ (i.e. when $\mathcal{L}_{p}=$ $\mathcal{L}_{2}=\mathcal{L}$ is the deformed CMS operator) this follows immediately from the Lemma. Indeed

$$
\left[\mathcal{L}_{2}, \mathcal{L}_{p}\right]=\sum_{v \in \mathcal{O}}\left[\mathcal{L}_{2}, \frac{\partial_{v}^{(p)}}{(v, v)}\right]=\sum_{v \in \mathcal{O}} \sum_{\alpha \in R^{+}} m_{\alpha} \frac{<\alpha, \alpha>}{<v, v>} \varphi_{\alpha}\left(\partial_{v}^{(p)}-\partial_{s<\alpha>v}^{(p)}\right),
$$

which obviously is identically zero.

To prove that these operators commute for any $p, q$ we borrow the idea from T. Oshima's paper [25]. Consider an involution $\sigma$ on the space of all differential operators on $V$ corresponding to the change $x \rightarrow-x$ and the standard anti-involution $*$ : operator $L^{*}$ is a formal adjoint to $L$. We have $\left[L_{1}^{\sigma}, L_{2}^{\sigma}\right]=\left[L_{1}, L_{2}\right]^{\sigma}$ and $\left[L_{1}^{*}, L_{2}^{*}\right]=-\left[L_{1}, L_{2}\right]^{*}$. Our operators $\mathcal{L}_{p}$ have the following properties with respect to these involutions: $\mathcal{L}_{p}^{*}=\mathcal{L}_{p}^{\sigma}=(-1)^{p} \mathcal{L}_{p}$.

Now let us consider the commutator $C=\left[\mathcal{L}_{p}, \mathcal{L}_{q}\right]$. By Jacobi identity $\left[C, \mathcal{L}_{2}\right]=0$, so we can use Berezin's lemma [26] which says that in such a case the highest symbol of $C$ must be polynomial in $x$. Since in our case it must also be periodic this implies that the highest symbol is constant. We claim that it is actually zero. Indeed $C^{*}=\left[\mathcal{L}_{p}, \mathcal{L}_{q}\right]^{*}=-\left[\mathcal{L}_{p}^{*}, \mathcal{L}_{q}^{*}\right]=$ $-\left[\mathcal{L}_{p}^{\sigma}, \mathcal{L}_{q}^{\sigma}\right]=-\left[\mathcal{L}_{p}, \mathcal{L}_{q}\right]^{\sigma}=-C^{\sigma}$, so $C^{*}=-C^{\sigma}$. Looking at the highest symbol in this relation we see that it must be zero. This completes the proof of the Theorem 1.

Notice that as follows from the formula for the integrals in the $B C_{n, m}$ case all the integrals $\mathcal{L}_{p}$ with odd $p$ are actually vanish, so in that case we will consider only even $p$.

Recall now that the quantum system in $\mathbf{R}^{n}$ is called integrable if it has at least $n$ commuting independent quantum integrals.

Corollary. Deformed CMS problems (4), (5) related to the classical generalized root systems are integrable. The same is true for their rational limits. 
To have the integrals in the rational limit one should replace in the formulas of this section $\sinh z$ by $z$ and $\operatorname{coth} z$ by $z^{-1}$, so that $f_{\alpha}(x)=$ $(\alpha, x)^{-1}, \varphi_{\alpha}(x)=-(\alpha, x)^{-2}$.

\section{Algebra $\Lambda_{R, B}$ And Harish-Chandra homomorphism.}

Let $R \subset V$ be a classical generalized root system and $(R, m, B)$ be its admissible deformation described in the section 1 . Let us introduce the corresponding algebra $\Lambda_{R, B}^{\omega}$ as the algebra of polynomial functions $p(x)$ on $V$, which satisfy the following properties (cf. [22]):

1) $p(x)$ are invariant with respect to Weyl group $W_{0}$, corresponding to the real roots of the system;

2) $p(x+\omega \alpha) \equiv p(x-\omega \alpha)$ on the hyperplane $(\alpha, x)=0$ for any imaginary root $\alpha$, where $(\alpha, x)$ is the deformed scalar product determined by $B$.

In the limit $\omega \rightarrow 0$ we have the algebra $\Lambda_{R, B}^{0}$ of $W_{0}$-invariant polynomials with the properties 1) and

$2)^{0} \partial_{\alpha} p(x) \equiv 0$ on the hyperplane $(\alpha, x)=0$ for any imaginary root $\alpha \in R$.

One can consider this algebra also as a subalgebra of polynomial functions on $V^{*}$ satisfying the same relation 2$)^{0}$ where $(\alpha, x)$ is understood as pairing between vector and covector and $\partial_{\alpha}$ is defined using the deformed form $B$. Below we will be using this realization.

Since the algebras $\Lambda_{R, B}^{\omega}$ are obviously isomorphic for all $\omega \neq 0$ we will assume later on that $\omega=1 / 2$ considering only algebras $\Lambda_{R, B}=\Lambda_{R, B}^{1 / 2}$ and $\Lambda_{R, B}^{0}$. We are going to show that for generic $k$ these two algebras are actually isomorphic to the algebras generated by the quantum integrals of the deformed CMS problems from the previous section in trigonometric and rational case respectively.

Remark. We should mention that in the case when all the multiplicities are integer the algebra of quantum integrals is actually much bigger and is called algebra of quasi-invariants, see [11], [23]). For example when $k=1$ the quasi-invariants are polynomials satisfying the property 2$)^{0}$ for all roots, but no symmetry is imposed (see [32] for the latest results in this direction).

It is obvious that the highest order component of any polynomial $P \in$ $\Lambda_{R, B}$ belongs to the algebra $\Lambda_{R, B}^{0}$. More subtle question is whether for any homogeneous $Q \in \Lambda_{R, B}^{0}$ there exists $P \in \Lambda_{R, B}$ such that $Q$ is the highest term of $P$. We will show that at least for generic values of the deformation parameter $k$ this is true, which means that $\Lambda_{R, B}^{0}$ is the associated graded algebra for $\Lambda_{R, B}$.

We are going now to describe the algebras $\Lambda_{R, B}^{0}$ more explicitly. Let us start with the type $A(n-1, m-1)$. The corresponding algebra can be realized as the following algebra $\Lambda_{n, m ; k}^{0} \subset \mathbf{C}\left[V^{*}\right]=\mathbf{C}\left[x_{1}, \ldots, x_{n}, y_{1}, \ldots, y_{m}\right]$ consisting of the polynomials $f\left(x_{1}, \ldots, x_{n}, y_{1}, \ldots, y_{m}\right)$ which are symmetric 
in $x_{1}, \ldots, x_{n}$ and $y_{1}, \ldots, y_{m}$ separately and satisfy the conditions

$$
\left(\frac{\partial}{\partial x_{i}}-k \frac{\partial}{\partial y_{j}}\right) f \equiv 0
$$

on each hyperplane $x_{i}-y_{j}=0$ for $i=1, \ldots, n$ and $j=1, \ldots, m$.

It is very easy to check that the deformed Newton sums

$$
p_{r}(x, y, k)=\sum_{i=1}^{n} x_{i}^{r}+\frac{1}{k} \sum_{j=1}^{m} y_{j}^{r}
$$

belong to $\Lambda_{n, m ; k}^{0}$ for all nonnegative integers $r$.

Theorem 2. If $k$ is not a positive rational number then the algebra $\Lambda_{n, m ; k}^{0}$ is generated by the deformed Newton polynomials $p_{r}(x, y, k), r \in \mathbf{Z}_{+}$.

Notice that for special values of $k$ this is not true. For example, if $k=1$ the deformed Newton sums generate the algebra of symmetric polynomials in $n+m$ variables, while $\Lambda_{n, m ; k}^{0}$ is a much bigger algebra containing for example $p=\prod\left(x_{i}-y_{j}\right)^{3}$.

To prove the Theorem let us recall that the partition $\lambda$ of a natural number $N$ is a decreasing sequence of non-negative integers $\lambda_{1} \geq \lambda_{2} \geq \lambda_{3} \geq \ldots$ such that only a finite number of them are non-zero and their sum is equal to $N$. This sum $\lambda_{1}+\lambda_{2}+\lambda_{3}+\ldots$ is usually denoted as $|\lambda|$. To each partition one can relate a Young diagram with $N$ squares in a natural way (see e.g. [24]).

Proposition 2. If $k$ is not a positive rational then the dimension of the homogeneous component $\Lambda_{n, m ; k}^{0}$ of degree $N$ is less or equal than the number of partitions $\lambda$ of $N$ such that $\lambda_{n+1} \leq m$.

Notice that the corresponding Young diagrams are precisely the ones contained in the fat $(n, m)$-hook (see Fig. 1).

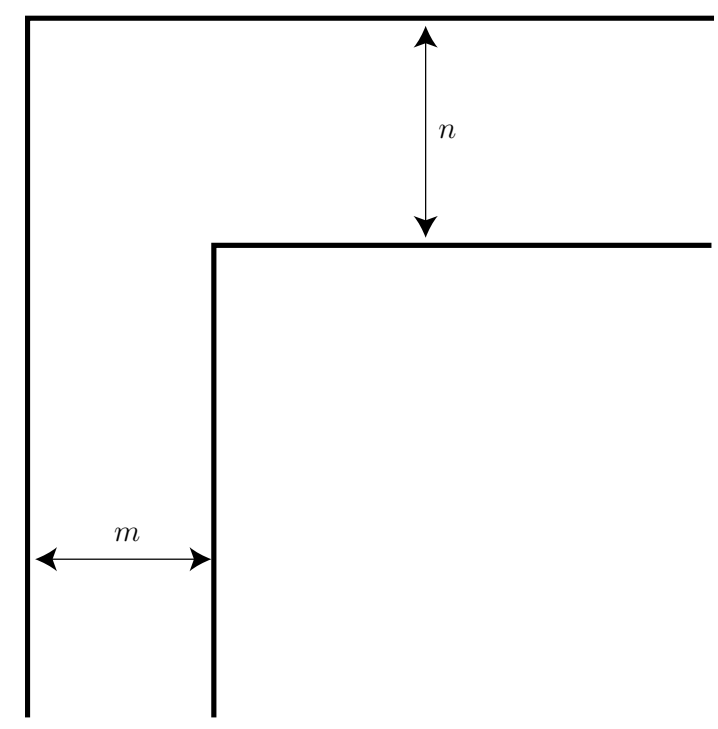

Fig. 1: Fat hook 
Denote by $D_{N}(n, m)$ the number of such partitions (or diagrams in this fat hook). Let $I=\left(i_{1}, i_{2}, \ldots, i_{n}\right)$ and $J=\left(j_{1}, j_{2}, \ldots, j_{m}\right)$ be some sequences (at the beginning, unordered) of nonnegative integers such that $\sum_{r=1}^{n} i_{r}+$ $\sum_{s=1}^{m} j_{s}=N$. Let $N(J)$ be the number of nonzero elements of $J, M(I, J)$ be the number of elements of $I$ which are greater or equal to $N(J)$. Define the sets

$$
E_{\text {reg }}=\{(I, J) \mid M(I, J)=n\}, \quad E_{\text {nreg }}=\{(I, J) \mid M(I, J)<n\} .
$$

The pairs from $E_{\text {reg }}$ will be called regular, otherwise - irregular.

Let us prescribe to each pair $(I, J)$ a variable $C(I, J)$ in such a way that $C(I, J)=C(\sigma(I), \tau(J)), \sigma \in S_{n}, \tau \in S_{m}$ is the same for all orderings of $I$ and $J$. It is easy to see that the number of different $C(I, J),(I, J) \in E_{\text {reg }}$ is equal to $D_{N}(n, m)$. For any sequence $I$ let us rewrite the elements of $I$ in non-increasing order and denote this sequence $I^{+}$. Choose some integers $1 \leq r \leq n$ and $1 \leq s \leq m$ and $1 \leq p \leq N$ and consider the equations

$$
\sum_{i+j=p}(i-k j) C(I, J)=0
$$

where $i$ occupies the $r$-th place in $I, j$ occupies the $s$-th place in $J$ and all other elements of $I, J$ are fixed. All such equations form the system of linear equations on $C(I, J)$, which has the following meaning. Let $f$ be a homogeneous polynomial of degree $N$, then the system (22) is nothing else but the condition 2$)^{0}$ for the coefficients of $f \in \Lambda_{n, m ; k}^{0}$.

Thus to prove the Proposition it is enough to show that every irregular $C(I, J),(I, J) \in E_{\text {nreg }}$ can be expressed from the system (22) as a linear combination of $C(I, J),(I, J) \in E_{\text {reg }}$.

Let us first prove this statement for $n=1$. We will use the induction with respect to the following total order on $E_{\text {nreg }}$. Let $(I, J),(K, L) \in E_{\text {nreg }}$. We will say that $(I, J)<(K, L)$ if for the corresponding ordered sets $\left(i, j_{1}^{+} \ldots j_{m}^{+}\right.$ and $\left(k, l_{1}^{+} \ldots l_{m}^{+}\right)$either $N(J)<N(L)$ or $N(J)=N(L)$ and for $q=\min \{i, k\}$ $\left(j_{m}^{+}, \ldots, j_{m-q}^{+}, i\right)<\left(l_{m}^{+}, \ldots, l_{m-q}^{+}, k\right)$ in the lexicographic order.

If $N(J)=1$ then we have

$k j C(0, j)+(-1+(j-1) k) C(1, j-1)+(-2+(j-2) k) C(2, j-2)+\cdots=0$.

So we have for $k \neq 0$ that $C(0, j) \in \operatorname{Span}\left\{C(i, j),(i, j) \in I_{\text {reg }}\right\}$

For general $N(J)>1$ take any $(I, J) \in E_{\text {nreg }}$ with an ordered $J=J^{+}$: $\left(i, j_{1}, j_{2}, \ldots\right)$. Consider the equation (22) corresponding to $r=1, s=$ $N(J)-i, p=i+j_{s}$. One can check that $(I, J)$ is the largest pair with respect to our order among all irregular pairs $(K, L)$ corresponding to $C(K, L)$ entering the equation with non-zero coefficients. Since the coefficient at $C(I, J)$ is $-i+k j_{s} \neq 0$ (because $k$ is not a positive rational) we can express $C(I, J)$ as a linear combination of lower variables. This proves Proposition for $n=1$.

For general $n$ we can use the induction in $M(I, J)$. If $M(I, J)=1$ we can use the previous arguments. Assume now that $M(I, J)>1$. Consider one index $r$, for which $i_{r}<N(J)$ and apply previous arguments to express 
$C(I, J)$ as a linear combination of $C\left(I^{*}, J^{*}\right)$ with $\left(I^{*}, J^{*}\right)$ such that $i_{r}^{*} \geq$ $N\left(J^{*}\right)$. According to inductive hypothesis we can express $C\left(I^{*}, J^{*}\right)$ as a linear combination $C\left(I^{* *}, J^{* *}\right)$ where $\left(I^{* *}, J^{* *}\right) \in I_{\text {reg }}$. Proposition 2 is proved.

Now let us prove the Theorem. Let us denote by $\mathcal{N}_{n, m ; k}$ the algebra generated by the deformed Newton sums (21). As we have already mentioned $\mathcal{N}_{n, m ; k} \subset \Lambda_{n, m ; k}^{0}$. To show that $\mathcal{N}_{n, m ; k}=\Lambda_{n, m ; k}^{0}$ it is enough to prove that the dimension of the homogeneous component of degree $N$ of $\mathcal{N}_{n, m ; k}$ is not less than $D_{N}(n, m)$. To produce enough independent polynomials we will use the theory of Jack polynomials (see e.g. [24]).

Let $\Lambda$ be algebra of symmetric functions in infinite number of variables $z_{1}, z_{2}, \ldots$ and $p_{r}(z)=z_{1}^{r}+z_{2}^{r}+\ldots$ be the power sum, $P_{\lambda}(z, \theta)$ be Jack polynomial depending of partition $\lambda$ (see [24] ). Consider a homomorphism $\phi$ from $\Lambda$ to $\Lambda_{n, m ; k}^{0}$ such, that

$$
\phi\left(p_{r}(z)\right)=p_{r}(x, y, k) .
$$

Such a homomorphism was first used by Kerov, Okounkov and Olshanski in [27]. The image of the Jack polynomials under this homomorphism sometimes is called super-Jack polynomials (see e.g. [28]).

One can show using some results from [24] (see formulas (7.9') and (10.19) from Chapter 6) that for $\theta=-k$

$$
\phi\left(P_{\lambda}(z, \theta)\right)=\sum_{\mu \subset \lambda} b_{\lambda / \mu}(\theta) P_{\mu}(x, \theta) P_{\lambda^{\prime} / \mu^{\prime}}\left(y, \theta^{-1}\right)
$$

where $b_{\lambda / \mu}$ is some rational function of $\theta$ with poles in non-positive rational numbers. Since $\theta=-k$ is not such a number by assumption these superJack polynomials are well-defined.

From the formula (23) it follows that the leading term in lexicographic order of $\phi\left(P_{\lambda}(z, \theta)\right)$ has a form

$$
x_{1}^{\lambda_{1}} \ldots x_{n}^{\lambda_{n}} y_{1}^{<\lambda_{1}^{\prime}-n>} \ldots y_{m}^{<\lambda_{m}^{\prime}-n>}
$$

where $\lambda^{\prime}$ is the partition conjugate to $\lambda$ and $\langle x\rangle=\frac{x+|x|}{2}=\max (0, x)$. From the definition $\phi\left(P_{\lambda}(z, \theta)\right) \in \mathcal{N}_{n, m ; k}$. It is clear that all these polynomials corresponding to the diagrams contained in the fat hook are linearly independent. This completes the proof of Theorem 2.

Remark. The relation with the theory of Jack polynomials is actually much deeper. We discuss this in detail in our paper [38] (see also [13], [14]).

As a corollary we can give a formula for Poincare series

$$
P_{n, m}(t)=\oplus_{i} \operatorname{dim}\left(\Lambda_{n, m ; k}^{0}\right)^{(i)}
$$

of the algebra $\Lambda_{n, m ; k}^{0}$ for generic $k$. 
Theorem 3. Poincare series of the algebra $\Lambda_{n, m ; k}^{0}$ for generic $k$ has the following form

$$
P_{n, m}(t)=\frac{1}{(1-t)\left(1-t^{2}\right) \ldots\left(1-t^{n}\right)}\left[1+\sum_{i=1}^{m} \frac{t^{i(n+1)}}{(1-t)\left(1-t^{2}\right) \ldots\left(1-t^{i}\right)}\right] .
$$

Proof. From Theorem 2 it follows that the corresponding Poincare series is the sum of $t^{|\lambda|}$ over all partitions $\lambda$ which fit into fat $(n, m)$ hook:

$$
P_{n, m}(t)=\sum_{\lambda_{n+1} \leq m} t^{|\lambda|}=\sum_{\lambda_{n+1}=0} t^{|\lambda|}+\sum_{\lambda_{n+1}=1} t^{|\lambda|}+\cdots+\sum_{\lambda_{n+1}=m} t^{|\lambda|},
$$

where $|\lambda|=\lambda_{1}+\lambda_{2}+\ldots+\lambda_{N}$. It is easy to see that

$$
\sum_{\lambda_{n+1}=i} t^{|\lambda|}=\sum_{\mu_{n+1}=0} \sum_{\nu_{i+1}=0} t^{i(n+1)+|\mu|+|\nu|}=t^{i(n+1)} \sum_{\mu_{n+1}=0} t^{|\mu|} \sum_{\nu_{i+1}=0} t^{|\nu|} .
$$

Since

$$
\sum_{\mu_{n+1}=0} t^{|\mu|}=\frac{1}{(1-t)\left(1-t^{2}\right) \ldots\left(1-t^{n}\right)}, \quad \sum_{\nu_{i+1}=0} t^{|\nu|}=\frac{1}{(1-t)\left(1-t^{2}\right) \ldots\left(1-t^{i}\right)}
$$

we arrive at the formula (24).

Remark. Another (recurrent) formula for the generating function of the Young diagrams which fit into fat hook was found recently by Orellana and Zabrocki [29] in relation with the theory of the Schur functions and characters of Lie superalgebras.

Notice that the symmetry between $n$ and $m$ is not obvious from our formula (24) and leads to some identities which might be interesting.

For $R=B C(n, m)$ the algebra $\Lambda_{R, B}^{0}$ is related to $\Lambda_{n, m ; k}^{0}$ in a very simple way: it is easy to check from the definition that it consists of the polynomials $p\left(x_{1}^{2}, x_{2}^{2}, \ldots, y_{1}^{2}, y_{2}^{2}, \ldots, y_{m}^{2}\right)$ where $p$ belong to $\Lambda_{n, m ; k}^{0}$.

Corollary. Poincare series $P_{n, m}^{B C}(t)$ of the algebra $\Lambda_{R, B}^{0}$ for generalized system $R$ of type $B C(n, m)$ and generic values of the deformation parameter has the following form

$$
P_{n, m}^{B C}(t)=P_{n, m}\left(t^{2}\right)
$$

where $P_{n, m}(t)$ is given by the formula $(24)$.

Let us discuss now the Harish-Chandra homomorphism. Let $R \in V$ be a generalized root system and $R^{+}$be a set of positive roots. Let us denote by $D\left[R^{-}\right]$the algebra of differential operators on $V^{*}$ with coefficients in $\mathbf{C}\left[e^{-\alpha},\left(e^{-\alpha}-1\right)^{-1}\right]$, where $\alpha \in R^{+}$.

The Harish-Chandra homomorphism $\varphi: D\left[R^{-}\right] \longrightarrow D$, where $D$ is the algebra of differential operators on $V^{*}$ with constant coefficients, is uniquely determined by the condition $\varphi\left(e^{-\alpha}\right)=0$. The algebra $D$ is isomorphic to the algebra of polynomial functions on the space $V^{*}$.

Let now $R$ be a classical generalized root systems and consider the algebra $Q_{R, m, B}$ generated by the quantum integrals $L_{s}$ of the corresponding 
deformed CMS problem (10):

$$
L_{s}=\hat{\psi}_{0} \circ \mathcal{L}_{s} \circ \hat{\psi}_{0}^{-1}
$$

where $\hat{\psi}_{0}$ is the multiplication operator by the function $\hat{\psi}_{0}=\prod_{\alpha \in R_{+}} \sin ^{-m_{\alpha}}(\alpha, x)$ and $\mathcal{L}_{s}$ given by (18). It is easy to check that all the operators $L_{s}$ belong to the algebra $D\left[R^{-}\right]$, so $Q_{R, m, B}$ is a subalgebra in $D\left[R^{-}\right]$.

Theorem 4. For generic values of the deformation parameter the HarishChandra homomorphism maps the algebra of quantum integrals of the deformed $C M S$ problems $Q_{R, m, B}$ onto the algebra $\Lambda_{R, B}$. In the rational limit the same is true for the algebra $\Lambda_{R, B}^{0}$.

Proof. We will identify $V$ and $V^{*}$ using the form $B$. Take $\lambda \in V$ and define $x_{v}^{p}(\lambda)=e^{-(\lambda, x)} \varphi\left(\partial_{v}^{(p)}\right) e^{(\lambda, x)}$. From (17) we have the following recurrent relations

$$
x_{v}^{(p)}(\lambda)=(\lambda, v) x_{v}^{(p-1)}(\lambda)-\frac{1}{2} \sum_{\alpha \in R^{+}} m_{\alpha}(\alpha, v)\left(x_{v}^{(p-1)}(\lambda)-x_{s<\alpha>(v)}^{(p-1)}(\lambda)\right)
$$

which can be rewritten as

$$
x_{v}^{(p)}(\lambda)=(\lambda-\rho, v) x_{v}^{(p-1)}(\lambda)+\frac{1}{2} \sum_{\alpha \in R^{+}} m_{\alpha}(\alpha, v) x_{s<\alpha>(v)}^{(p-1)}(\lambda) .
$$

The shifted functions $y_{v}^{(p)}(\lambda)=x_{v}^{(p)}(\lambda+\rho)$ satisfy the relations

$$
y_{v}^{(p)}(\lambda)=(\lambda, v) y_{v}^{(p-1)}(\lambda)+\frac{1}{2} \sum_{\alpha \in R^{+}} m_{\alpha}(\alpha, v) y_{s_{<\alpha>(v)}^{(p-1)}}^{(\lambda) .}
$$

It is easy to see that the the image $\mathcal{Z}_{p}=\varphi\left(L_{p}\right)$ of the quantum integrals $L_{p}$ under Harish-Chandra homomorphism has the form

$$
\mathcal{Z}_{p}=\sum_{v \in \mathcal{O}} \frac{y_{v}^{(p)}}{(v, v)}
$$

Let $(\lambda, \gamma)=0$, where $\gamma \in R_{i m}$ is an imaginary root. We should prove that

$$
\mathcal{Z}_{p}\left(\lambda-\frac{\gamma}{2}\right)=\mathcal{Z}_{p}\left(\lambda+\frac{\gamma}{2}\right)
$$

We will prove this for the root system of type $A_{n, m}$, the case of $B C_{n, m}$ root system is very similar.

Without loss of generality we can assume that $\gamma=e_{n}-e_{n+1}$. Let us introduce $y_{v}^{(p)-}(\lambda)=y_{v}^{(p)}\left(\lambda-\frac{\gamma}{2}\right), y_{v}^{(p)+}(\lambda)=y_{v}^{(p)}\left(\lambda+\frac{\gamma}{2}\right)$. We have the following recurrent relations

$$
\begin{aligned}
& y_{v}^{(p)-}(\lambda)=\left(\lambda-\frac{1}{2} \gamma, v\right) y_{v}^{(p-1)-}(\lambda)+\frac{1}{2} \sum_{\alpha \in R^{+}} m_{\alpha}(\alpha, v) y_{s_{<\alpha>}(v)}^{(p-1)-}(\lambda) \\
& y_{v}^{(p)+}(\lambda)=\left(\lambda+\frac{1}{2} \gamma, v\right) y_{v}^{(p-1)+}(\lambda)+\frac{1}{2} \sum_{\alpha \in R^{+}} m_{\alpha}(\alpha, v) y_{s_{<\alpha>(v)}}^{(p-1)+}(\lambda)
\end{aligned}
$$


Let us denote by $v=e_{n}, u=s_{<\gamma>}(v)=e_{n+1}$ and introduce $y_{v, u}^{(p) \pm}(\lambda)$ as

$$
y_{v, u}^{(p) \pm}(\lambda)=\left((u, u)^{-1}+(v, v)^{-1}\right)^{-1}\left[(u, u)^{-1} y_{u}^{(p) \pm}+(v, v)^{-1} y_{v}^{(p) \pm}\right] .
$$

Lemma. On the hyperplane $(\lambda, \gamma)=0$ the following relations hold:

$$
\text { 1) } y_{v, u}^{(p) \pm}(\lambda)=(\lambda, v) y_{v, u}^{(p-1) \pm}(\lambda)+\frac{1}{2} \sum_{s<\alpha>(v) \neq u, v} m_{\alpha}(\alpha, v) y_{s_{<\alpha>}(v)}^{(p-1) \pm}(\lambda),
$$

2) $y_{v}^{(p)-}(\lambda)=y_{u}^{(p)-}(\lambda)$.

Proof is by induction and based on the following fact, which can be easily checked directly: if $s_{<\alpha>}(v)=s_{<\beta>}(u)$ then

$$
\left((u, u)^{-1}+(v, v)^{-1}\right)^{-1}\left[\frac{m_{\alpha}(\alpha, v)}{(v, v)}+\frac{m_{\beta}(\beta, u)}{(u, u)}\right]=m_{\alpha}(\alpha, v)=m_{\beta}(\beta, v) .
$$

Now let $w=e_{s}, w \neq u, v$ then one can check that

$$
\left(m_{\alpha}(\alpha, w) y_{v}^{(p-1)+}+m_{\beta}(\beta, w) y_{u}^{(p-1)+}=\left(m_{\alpha}(\alpha, w)+m_{\beta}(\beta, w)\right) y_{v, u}^{(p-1)+}\right.
$$

where $s_{<\alpha>}(v)=s_{<\beta>}(u)=w$. Notice that the last relation determines $\alpha$ and $\beta$ uniquely in our case. Using this one can show that

$y_{w}^{(p) \pm}=(\lambda, w) y_{w}^{(p-1) \pm}+\frac{1}{2}\left(m_{\alpha}(\alpha, w)+m_{\beta}(\beta, w)\right) y_{v, u}^{(p-1) \pm}+\frac{1}{2} \sum_{s_{<\delta}>(w) \neq u, v} m_{\delta}(\delta, w) y_{s_{<\delta>(w)}}^{(p-1) \pm}$

provided $(\lambda, \gamma)=0$

From the relations $(26),(27)$ it follows that $y_{w}^{(p)+}=y_{w}^{(p)-}, y_{v, u}^{(p)-}=y_{v, u}^{(p)+}$ on the hyperplane $(\lambda, \gamma)=0$, which imply the relation (25).

Note that the highest term of $\mathcal{Z}_{r}(\lambda)$ is

$$
\lambda_{1}^{r}+\cdots+\lambda_{n}^{r}+k^{r-1}\left(\lambda_{n+1}^{r}+\cdots+\lambda_{n+m}^{r}\right) .
$$

Now from Theorem 2 it follows that for generic $k$ homomorphism $\varphi$ is surjective. The fact that it is injective is obvious. This completes the proof in trigonometric case, rational case easily follows. In fact $\omega$ in the definition of $\Lambda_{R, B}^{\omega}$ and $\omega$ in the limiting procedure from trigonometric to rational case could be identified.

Remark. Notice that we have proved that the image of Harish-Chandra homomorphism belong to the algebra $\Lambda_{R, B}$ for all values of the parameter $k$. The condition that $k$ is generic is used only to claim that the image coincides with this algebra.

As a corollary we have the following statement which is probably true for all generalized root systems and all values of deformation parameter.

Proposition 3. For the classical generalized root systems and generic values of the deformation parameter the algebra $\Lambda_{R, B}^{0}$ is the associated graded algebra for $\Lambda_{R, B}$. 
In $A(n, m)$ case we can give an explicit formula for the generators of the algebra $\Lambda_{R, B}$ :

$$
Y_{r}(\lambda)=\sum_{i=1}^{n} B_{r}\left(\lambda_{i}+1 / 2\right)+k^{r-1} \sum_{j=1}^{m} B_{r}\left(\lambda_{j+n}+1 / 2\right),
$$

where $B_{r}(x)$ are the classical Bernoulli polynomials. One can easily check using the relation $B_{r}(x+1)-B_{r}(x)=r x^{r-1}$ that $Y_{r}$ satisfy the relations (25) and have the highest term (28).

We finish this section with the following

Theorem 5. Algebra $\Lambda_{n, m ; k}^{0}$ is finitely generated if and only if $k$ is not a negative rational number of the form $-\frac{s}{r}$, where $1 \leq r \leq n, 1 \leq s \leq m$.

Consider the subalgebra $P(k)=\mathbf{C}\left[p_{1}, \ldots p_{n+m}\right]$ generated by the first $n+m$ deformed Newton sums (21). We need the following result about common zeros of these polynomials (cf. Proposition 1 in [32]).

Proposition 4. Consider the following system of algebraic equations

$$
\left\{\begin{array}{r}
x_{1}+x_{2}+\cdots+x_{n}+k^{-1}\left(x_{n+1}+x_{n+2}+\cdots+x_{n+m}\right)=0 \\
x_{1}^{2}+x_{2}^{2}+\cdots+x_{n}^{2}+k^{-1}\left(x_{n+1}^{2}+x_{n+2}^{2}+\cdots+x_{n+m}^{2}\right)=0 \\
\\
x_{1}^{n+m}+x_{2}^{n+m}+\cdots+x_{n}^{n+m}+k^{-1}\left(x_{n+1}^{n+m}+x_{n+2}^{n+m}+\cdots+x_{n+m}^{n+m}\right)=0
\end{array}\right.
$$

If parameter $k$ is not a negative rational number of the form $-\frac{s}{r}$, where $1 \leq r \leq n, 1 \leq s \leq m$ then the system has only trivial (zero) solution in $\mathbf{C}^{n+m}$. Converse statement is also true.

To prove this suppose that the system has a nontrivial solution $x_{1}, \ldots, x_{n+m}$. We can assume that $x_{i} \neq 0$ for all $i=1, \ldots, n+m$. Let us re-group the set $X=\left\{x_{1}, x_{2}, \ldots, x_{n+m}\right\} \subset \mathbf{C}$ identifying equal $x_{i}$ 's as $\left\{z_{1}, \ldots, z_{p}\right\}, p \leq n+m$, where all $z_{j}$ are different. Multiplicity of $z_{j}$ is a pair $\left(r_{j}, s_{j}\right)$, where $r_{j}$ shows how many times $z_{j}$ enters the set $\left\{x_{1}, x_{2}, \ldots, x_{n}\right\}$ and $s_{j}$ is the same for the rest of the set $X$. For the numbers $z_{j}, \quad 1 \leq j \leq p$ we have the system

$$
\sum_{j=1}^{p} a_{j} z_{j}^{i}=0, \quad i=1, \ldots, n+m,
$$

where $a_{j}=r_{j}+k^{-1} s_{j}$. Consider the first $p$ of these equations as the linear system on $a_{j}$. Its determinant is of Vandermonde type and is not zero since all $z_{j}$ are different and non-zero. Hence all $a_{j}$ must be zero which may happen only if $k=-\frac{s}{r}$ for some $1 \leq r \leq n, 1 \leq s \leq m$. The converse statement is obvious: if $k=-\frac{s}{r}$ then we can take $x_{1}=x_{2}=\cdots=x_{r}=z=$ $x_{n+1}=\cdots=x_{n+s}$ and other $x_{i}$ being zero to have the non-trivial solutions of the system with arbitrary $z$.

From Proposition 4 it follows that for $k \neq-\frac{s}{r}$ the algebra of all polynomials on $V$ is a finitely generated module over subalgebra $P(k)$. By a general result from commutative algebra (see e.g. Proposition 7.8 from [30]) this implies that $\Lambda_{n, m ; k}^{0}$ is finitely generated. 
Now suppose that $k=-\frac{s}{r}$ for some $1 \leq r \leq n, 1 \leq s \leq m$. Consider the following homomorphism

$$
\phi_{r, s}: \Lambda_{n, m ; k}^{0} \rightarrow \Lambda_{1,1 ;-1}^{0}
$$

by sending a polynomial $f\left(x_{1}, \ldots, x_{n}, y_{1}, \ldots, y_{m}\right)$ into

$$
\hat{f}(x, y)=f(x, x, \ldots, x, 0, \ldots, 0, y, y, \ldots, y, 0, \ldots, 0),
$$

where $x$ is repeated $r$ times and $y$ is repeated $s$ times. One can easily check that if $f$ is in $\Lambda_{n, m ; k}^{0}$ with this particular $k$ then $\hat{f}(x, y)$ satisfies the condition $\left(\partial_{x}+\partial_{y}\right) \hat{f}=0$ when $x=y$, i.e. $\hat{f}$ belongs to $\Lambda_{1,1 ;-1}^{0}$. The last algebra is actually very simple: it consists of the polynomials which are constant on the line $x=y$ and thus have the form $c+q(x, y)(x-y)$ with arbitrary polynomial $q$. It is easy to see that this algebra is not finitely generated. Since it is a homomorphic image of the algebra $\Lambda_{n, m ; k}^{0}$ this implies that the algebra $\Lambda_{n, m ; k}^{0}$ with $k=-\frac{s}{r}$ is also not finitely generated. Theorem 5 is proved.

Remark. The case $k=-1$ is actually very special: in that case the algebra $\Lambda_{n, m ; k}^{0}$ is known as algebra of supersymmetric polynomials and plays an important role in geometry (see e.g. [31], Chapter 3). Notice also that the special values of $k$ in the theorem 2 are positive rationals while in theorem 5 they are negative.

Corollary. For generic values of the deformation parameters the algebras $\Lambda_{R, B}^{0}$ and $\Lambda_{R, B}$ for the classical generalized root systems are finitely generated.

Indeed, from Proposition 3 it follows that it is enough to show that $\Lambda_{R, B}^{0}$ is finitely generated. For $A(n, m)$ type this follows directly from Theorem 5 , to prove this for $B C(n, m)$ one should replace in Theorem 5 all the coordinates by their squares.

An interesting question is whether the algebra $\Lambda_{n, m ; k}^{0}$ is free as a module over its polynomial subalgebra $P(k)$, i.e. has Cohen-Macaulay property. If this is true (which we believe to be so) then our formula (24) gives the degrees of its generators for generic values of $k$.

For example, when $m=1$ we have

$P_{n, 1}(t)=\frac{1}{(1-t)\left(1-t^{2}\right) \ldots\left(1-t^{n}\right)}\left[1+\frac{t^{n+1}}{(1-t)}\right]=\frac{1+t^{n+2}+t^{n+3}+\cdots+t^{2 n+1}}{(1-t)\left(1-t^{2}\right) \ldots\left(1-t^{n+1}\right)}$,

which shows that the generators should have the degrees $0, n+2, n+3, \ldots, 2 n+$ 1. The conjecture is that one can take the corresponding deformed Newton sums as such generators.

For $n=2$ this is in a good agreement with the results from [32], where the Cohen-Macaulay property for the rings of quasi-invariants related to $A(n, 1)$ and $B C(n, 1)$ is established (for any $n$ ) and the corresponding Poincare series are found (for $n=2$ ). 


\section{Generalizations: elliptic And difference Versions.}

The deformed quantum CMS systems we discussed have some natural generalizations. First of all if we replace in all the formulas for these operators the function $\frac{1}{\sin ^{2} z}$ by Weierstrass' elliptic function $\wp(z)$ we will have the deformed elliptic CMS operators.

For the generalized root system of type $A(n-1, m-1)$ we have

$$
\begin{aligned}
L_{A(n-1, m-1)}^{e l l}= & -\left(\frac{\partial^{2}}{\partial x_{1}{ }^{2}}+\cdots+\frac{\partial^{2}}{\partial x_{n}{ }^{2}}\right)-k\left(\frac{\partial^{2}}{\partial y_{1}{ }^{2}}+\cdots+\frac{\partial^{2}}{\partial y_{m}{ }^{2}}\right) \\
& +\sum_{i<j}^{n} 2 k(k+1) \wp\left(x_{i}-x_{j}\right)+\sum_{i<j}^{m} 2\left(k^{-1}+1\right) \wp\left(y_{i}-y_{j}\right) \\
& +\sum_{i=1}^{n} \sum_{j=1}^{m} 2(k+1) \wp\left(x_{i}-y_{j}\right) .
\end{aligned}
$$

For $B C(n, m)$ we can write a more general deformed Inozemtsev operator:

$$
\begin{aligned}
L_{B C(n, m)}^{e l l}= & -\left(\frac{\partial^{2}}{\partial x_{1}{ }^{2}}+\cdots+\frac{\partial^{2}}{\partial x_{n}{ }^{2}}\right)-k\left(\frac{\partial^{2}}{\partial y_{1}{ }^{2}}+\cdots+\frac{\partial^{2}}{\partial y_{m}{ }^{2}}\right) \\
& +\sum_{i<j}^{n} 2 k(k+1)\left(\wp\left(x_{i}-x_{j}\right)+\wp\left(x_{i}+x_{j}\right)\right)+\sum_{i<j}^{m} 2\left(k^{-1}+1\right)\left(\wp\left(y_{i}-y_{j}\right)+\wp\left(y_{i}+y_{j}\right)\right) \\
& +\sum_{i=1}^{n} \sum_{j=1}^{m} 2(k+1)\left(\wp\left(x_{i}-y_{j}\right)+\wp\left(x_{i}+y_{j}\right)\right) \\
& +\sum_{i=1}^{n} \sum_{l=0}^{3} q_{l}\left(q_{l}+1\right) \wp\left(x_{i}+\omega_{l}\right)+\sum_{j=1}^{m} \sum_{l=0}^{3} s_{l}\left(s_{l}+1\right) \wp\left(y_{j}+\omega_{l}\right)
\end{aligned}
$$

where $\omega_{0}=0, \omega_{l}, l=1,2,3$ are the half-periods of the corresponding elliptic curve and the 9 parameters $k, q_{l}, s_{l}, l=0,1,2,3$ satisfy the following 4 relations

$$
2 q_{l}+1=k\left(2 s_{l}+1\right)
$$

for all $l$.

We conjecture that the elliptic versions of the deformed CMS problems are integrable as well. For $m=1$ some results in this direction are found in [33],[34] (see also recent paper [35]).

Consider now the difference case. Let us introduce the following deformed Macdonald-Ruijsenaars operator. It depends on two parameters $t$ and $q$ and has the form

$$
D^{n, m}=\frac{1}{1-q} \sum_{i=1}^{n} A_{i} T_{q, x_{i}}+\frac{1}{1-t} \sum_{j=1}^{m} B_{j} T_{t, y_{j}}
$$


where

$$
A_{i}=\prod_{k \neq i}^{n} \frac{\left(x_{i}-t x_{k}\right)}{\left(x_{i}-x_{k}\right)} \prod_{j=1}^{m} \frac{\left(x_{i}-q y_{j}\right)}{\left(x_{i}-y_{j}\right)}, \quad B_{j}=\prod_{i=1}^{n} \frac{\left(y_{j}-t x_{i}\right)}{\left(y_{j}-x_{i}\right)} \prod_{l \neq j}^{m} \frac{\left(y_{j}-q y_{l}\right)}{\left(y_{j}-y_{l}\right)}
$$

and $T_{q, x_{i}}, T_{t, y_{j}}$ are the "shift operators":

$$
\begin{aligned}
& \left(T_{q, x_{i}} f\right)\left(x_{1}, \ldots, x_{i}, \ldots, x_{n}, y_{1}, \ldots, y_{m}\right)=f\left(x_{1}, \ldots, q x_{i}, \ldots, x_{n}, y_{1}, \ldots, y_{m}\right) \\
& \left(T_{t, y_{j}} f\right)\left(x_{1}, \ldots, x_{n}, y_{1}, \ldots, y_{j}, \ldots, y_{m}\right)=f\left(x_{1}, \ldots, x_{n}, y_{1}, \ldots, t y_{j}, \ldots, y_{m}\right) .
\end{aligned}
$$

For $m=1$ a similar operator was considered by O. Chalykh in [37]. We should mention that there is a small discrepancy between his and our formulas because of the misprint in the formula (7.3) in [37].

It is interesting that the form of the operator (33) is invariant under the simultaneous interchange of $q \leftrightarrow t$ and $x \leftrightarrow y$. In a way the deformed operator (33) is more symmetric than the original Macdonald-Ruijsenaars operator $[24],[36]$. In the differential case this duality corresponds to the invariance of the family of the deformed CM operators (4) under the interchange $x \leftrightarrow y$ and $k \leftrightarrow \frac{1}{k}$.

One can verify that the operator (33) can be rewritten in terms of the root system $R$ of type $A(n, m)$ as follows (notations are as in Section 3 )

$$
D^{R}=\sum_{v \in \mathcal{O}} \frac{1}{1-q^{(v, v)}}\left[\prod_{\alpha \in R,<\alpha, v>>0} \frac{1-t_{\alpha}^{(\alpha, v)} q^{-\alpha}}{1-q^{-\alpha}}\right] T_{v}
$$

where

$$
t_{\alpha}=q^{m_{\alpha}}, \quad q^{\alpha}(u)=q^{(\alpha, u)}, \quad\left(T_{v} f\right)(u)=f(u+v)
$$

and the relation with the previous formula is given by

$$
t=q^{k}, \quad x_{i}=q^{e_{i}}, \quad y_{j}=q^{e_{n+j}} .
$$

In this form the operator can be immediately generalized for any reduced generalized root system $B_{n, m}, C_{n, m}, D_{n, m}$ (but not for the general $B C(n, m$ ) case). We intend to discuss the properties of these deformed Macdonald operators in a separate paper.

\section{CONCLUDING REMARKS.}

We have constructed for each generalized root system a family of the deformed quantum CMS problems and proved their integrability for the classical series. The proof is effective but not conceptual. We present more conceptual proof for $A(n, m)$ series in our paper [38], where the algebraic varieties corresponding to the rings $\Lambda_{R, B}^{0}$ and relations with the theory of Jack polynomials are also discussed in more detail.

It is clear that these relations between the theory of Lie superalgebras and quantum integrable systems should be understood better. In particular, the spectral theory for the deformed CMS operators should be related to the representation theory of Lie superalgebras and spherical functions on the symmetric superspaces. We hope to come back to this problem soon. 


\section{ACKNOWLEDGEMENTs.}

We are grateful to O. Chalykh, A. Okounkov, G. Olshanski and especially to M. Feigin for useful discussions and helpful remarks.

This work was supported by EPSRC (grants GR/R70194/01 and GR/M69548).

The second author (A.P.V.) is grateful to IHES (Bures-sur-Yvette, France) for the hospitality in February 2003 when the final version of this paper was prepared.

\section{REFERENCES}

[1] F.Calogero Solution of the one-dimensional $N$-body problems with quadratic and/or inversely quadratic pair potentials. J. Math. Phys. 12, 1971, 419-436.

[2] B. Sutherland Exact results for a quantum many-body problem in one dimension. Phys. Rev. A 4, 1971, 2019-2021.

[3] M.A. Olshanetsky, A.M. Perelomov Quantum integrable systems related to Lie algebras. Phys. Rep. 94, 1983, 313-404.

[4] M.A.Olshanetsky, A.M.Perelomov Quantum systems related to root systems and radial parts of Laplace operators. Funct. Anal. Appl. 12, 1978, 121-128.

[5] F.A. Berezin, G.P. Pokhil, V.M. Finkelberg Schrödinger equation for a system of one-dimensional particles with point interaction. Vestnik MGU, No. 1, 1964, 21-28.

[6] S. Helgason Groups and Geometric Analysis. Academic Press, 1984.

[7] G. J. Heckman and E. M. Opdam, Root systems and hypergeometric functions I, Comp. Math. 64, 1987, 329-352

[8] G. J. Heckman, A remark on the Dunkl differential-difference operators, Progress in Math. 101, 1991, 181-191

[9] A.P. Veselov, M.V. Feigin, O.A. Chalykh New integrable deformations of quantum Calogero - Moser problem. Russian Math. Surveys 51, no.3, 1996, 185-186.

[10] O.A. Chalykh, M.V. Feigin, A.P. Veselov New integrable generalizations of CalogeroMoser quantum problem. J. Math. Phys 39 (2), 1998, 695-703.

[11] O.A. Chalykh, M.V. Feigin, A.P. Veselov Multidimensional Baker-Akhiezer Functions and Huygens' Principle. Commun. Math. Physics 206, 1999, 533-566.

[12] A.P. Veselov Deformations of the root systems and new solutions to generalized WDVV equations. Phys. Lett. A 261, 1999, 297-302.

[13] A.N. Sergeev Superanalogs of the Calogero operators and Jack polynomials. J. Nonlin. Math. Phys. 8, 2001, no. 1, 59-64.

[14] A.N. Sergeev Calogero operator and Lie superalgebras. Theor. Math. Phys. 131, no.3, $2002,747-764$.

[15] V. Serganova On generalization of root system. Commun. in Algebra 24(13), 1996, 4281-4299.

[16] N. Bourbaki Groupes et algèbres de Lie. Chap. VI, Masson, 1981.

[17] V.G. Kac Lie superalgebras. Adv. Math. 26, 1977, no.1, 8-96.

[18] Yu. Berest Private communication.

[19] A. Matsuo Integrable conections related to zonal spherical functions. Invent. Math. 110, 1992, 95-121.

[20] A.P. Veselov On generalisations of the Calogero-Moser-Sutherland quantum problem and WDVV equations. J. Math. Phys. 43, 2002, no. 11, 5675-5682.

[21] O.A. Chalykh, A.P.Veselov Locus configurations and $\vee$-systems. Physics Letters A 285, 2001, 339-349.

[22] O.A. Chalykh, A.P. Veselov Commutative rings of partial differential operators and Lie alrebras. Commun. Math. Phys. 126, 1990, 597-611. 
[23] M. Feigin and A. P. Veselov Quasi-invariants of Coxeter groups and m-harmonic polynomials. Int. Math. Research Notices, No. 10, 2002, 521-545.

[24] I. Macdonald Symmetric functions and Hall polynomials 2nd edition, Oxford Univ. Press, 1995.

[25] T. Oshima Completely integrable systems with a symmmetry in coordinates. Asian J. Math. 2., 1998, 935-955.

[26] F.A. Berezin Laplace operators on semisimple Lie groups. Proc. Moscow Math. Soc. 6, 1971, 371-463 (Russian).

[27] S. Kerov, A. Okounkov, G. Olshanski The boundary of the Young graph with Jack edge multipliers. Intern. Math. Res. Notices, 1998, no.4, 173-199.

[28] A. Okounkov On N-point correlations in the log-gas at rational temperature. hepth/9702001.

[29] R.C. Orellana, M. Zabrocki Some remarks on the characters of the general Lie superalgebra. math.CO/0008152.

[30] M. Atiyah, I.G. Macdonald Introduction to Commutative Algebra. Addison-Wesley, 1969.

[31] W. Fulton, P. Pragacz Schubert Varieties and Degeneracy Loci. Lect. Notes in Math. 1689, Springer, 1998.

[32] M. Feigin and A. P. Veselov Quasi-invariants and quantum integrals of the deformed Calogero-Moser systems. Preprint IHES M/03/13, February 2003.

[33] L.A. Khodarinova On quantum elliptic Calogero-Moser problem. Vestnik MGU, Ser.I Math. Mech., 1998, n.5, 16-19.

[34] L.A. Khodarinova, I.A. Prikhodsky On algebraic integrability of the deformed elliptic Calogero-Moser problem. J. Nonlin. Math. Phys. 8, 2001, no. 1, 50-53.

[35] O. Chalykh, P. Etingof, A. Oblomkov Generalized Lamé operators. math.QA/0212029.

[36] S.N.M. Ruijsenaars Complete integrability of relativistic Calogero-Moser systems ans elliptic functions identities. Comm. Math. Phys. 110, 1987, 191-213.

[37] O. Chalykh Macdonald polynomials and algebraic integrability. Adv. Math. 166, 2002, 193-259.

[38] A.N. Sergeev, A.P. Veselov Generalized discriminants, deformed quantum CalogeroMoser systems and Jack polynomials. In preparation. 